hep-th/0209136

SNBNCBS-2002

\title{
Abelian 2-form gauge theory: special features
}

\author{
R. P. Malik* \\ S. N. Bose National Centre for Basic Sciences, \\ Block-JD, Sector-III, Salt Lake, Calcutta-700 098, India
}

\begin{abstract}
It is shown that the four $(3+1)$-dimensional (4D) free Abelian 2-form gauge theory provides an example of (i) a class of field theoretical models for the Hodge theory, and (ii) a possible candidate for the quasi-topological field theory (q-TFT). Despite many striking similarities with some of the key topological features of the two $(1+1)$-dimensional (2D) free Abelian (and self-interacting non-Abelian) gauge theories, it turns out that the 4D free Abelian 2-form gauge theory is not an exact TFT. To corroborate this conclusion, some of the key issues are discussed. In particular, it is shown that the (anti-)BRST and (anti)co-BRST invariant quantities of the 4D 2-form Abelian gauge theory obey the recursion relations that are reminiscent of the exact TFTs but the Lagrangian density of this theory is not found to be able to be expressed as the sum of (anti-)BRST and (anti-)co-BRST exact quantities as is the case with the topological 2D free Abelian (and self-interacting non-Abelian) gauge theories.
\end{abstract}

*E-mail: malik@boson.bose.res.in 


\section{Introduction}

In recent years, there has been an upsurge of interest in the study of some of the key physical and mathematical issues associated with the 4D massless 2-form (antisymmetric) Abelian gauge theory which has already been proven to provide a dual description for the massless scalar field theory (see, e.g. [1-3]). Such an interest has been thriving for the past few years due to the relevance of the basic field (a second rank antisymmetric tensor $B_{\mu \nu}=-B_{\nu \mu}$ ) of this theory in the context of modern developments in the subject of (super)string theories, related issues of the extended objects, supergravity theories and non-commutative field theories. For instance, the antisymmetric potential $B_{\mu \nu}$ of this 2-form gauge theory appears very naturally in the supergravity multiplets [4] and in the excited states of the quantized (super)string theories (see, e.g., $[5,6]$ for details). The existence of this field is also crucial to the anomaly cancellation mechanism for the superstring theories and its presence provides an estimate for the dualities in the extended objects (see, e.g., $[5,6]$ ). This field has also turned up in an elegant way in the context of modern developments in the subject of noncommutative geometry [7]. Besides the above relevance and importance, the antisymmetric potential and the corresponding gauge theory have been an interesting topic of discussion for diverse and distinct reasons in various branches of theoretical physics, e.g., cosmic string theory, vortices in an incompressible and irrotational fluid, QCD, "hairs" on the black holes, etc. [8-11]. It is now quite well known that this 2-form antisymmetric $B_{\mu \nu}$ field generates an effective mass for the one-form Abelian gauge field $A_{\mu}$ in $4 \mathrm{D}$ through a topological coupling (i.e. celebrated $B \wedge F$ ) term where the gauge invariance and mass co-exist together without the presence of a residual scalar Higgs field [3,11,12]. This latter theory has been studied from various points of view because of its innate rich mathematical structure and physical relevance. In this connection, mention can be made of interesting discussions related to this theory such as Dirac bracket analyses [12-14], BFT Hamiltonian formulations [15], BRST quantization [16], duality considerations [17,18], etc.

The covariant canonical quantization of the massless 2-form gauge theory was first attempted in [19]. This aspect of quantization has been subsequently studied in a systematic manner by a host of authors in the framework of Becchi-Rouet-Stora-Tyutin (BRST) formalism [20-24]. It was observed that this theory possesses an interesting constraint structure and a naive gauge-fixing term (containing the antisymmetric tensor field) is found to be invariant under a secondary gauge transformation. This intrinsic property of the theory requires the introduction of ghosts for ghost fields so that the gauge-fixing can be achieved in a complete and systematic fashion for the purpose of BRST quantization. In all the above approaches [19-24], the generator of the BRST transformation (i.e. the nilpotent $\left(Q_{b}^{2}=0\right)$ and conserved $\left(\dot{Q}_{b}=0\right)$ BRST charge $\left.Q_{b}\right)$ turns out to define the BRST cohomology in the quantum Hilbert space of states. In the language of the differential geometry and cohomology, the analogue of the exterior derivative $d$ (with $d^{2}=0, d=d x^{\mu} \partial_{\mu}$ ) is none other than the BRST charge $Q_{b}$ connected with the (gauge) description of the 2- 
form massless gauge theory. In a recent paper [25], however, the usual BRST formalism with a single BRST charge $Q_{b}$ (i.e. the analogue of $d$ ) has been extended to include the symmetry generators (as conserved charges) $Q_{b}, Q_{d}, Q_{w}$ that produce local, covariant and continuous symmetry transformations for the Lagrangian density of the theory. The latter two symmetry transformations, generated by $Q_{d}$ and $Q_{w}$, correspond to the co-exterior derivative $\delta= \pm * d *$ (with $\delta^{2}=0$ ) and the Laplacian operator $\Delta=(d+\delta)^{2}=d \delta+\delta d$ where $*$ is the Hodge duality operation [26,27]. We christen the symmetry transformations on the fields corresponding to the analogue of $\delta$ as the dual(co)-BRST symmetry transformations and a bosonic symmetry (which is equal to the anti-commutators of nilpotent (anti-)BRST and (anti-)co-BRST symmetries) corresponds to the Laplacian operator $\Delta$. One can explain the existence of these symmetries in the language of the invariances of certain specific terms of the Lagrangian density of the theory which are closely connected with the cohomological operators. For instance, the (anti-)BRST transformations keep the kinetic energy term (or, more precisely, the curvature term $H=d B$ itself $^{\dagger}$ ) invariant. In contrast, it is the gauge-fixing term $\left(\delta B=\partial_{\mu} B^{\mu \nu} d x_{\nu}\right)$ that remains invariant under the (anti-)co-BRST transformations. The bosonic symmetry corresponding to the Laplacian operator leaves the ghost fields invariant (or ghost fields, at the most, transform by a vector gauge transformations). Together all the three symmetry transformations of the theory correspond to all the de Rham cohomological operators $(d, \delta, \Delta)$ of the differential geometry so that the analogue of the Hodge decomposition theorem (HDT) could be defined in the quantum Hilbert space of states in terms of the above conserved charges [25]. There exists a discrete symmetry in the theory (cf (2.4) below) that corresponds to the Hodge duality * operation of the differential geometry [25].

It would be nice to recall that, analogous to the 4D 2-form Abelian gauge theory, the free 2D Abelian $U(1)$ gauge theory [31-33,41,43], the 2D self-interacting non-Abelian gauge theory (where there is no interaction between gauge fields and matter fields) [33,34,41,42] and the 2D interacting $U(1)$ gauge theory (where there is an interaction between the $U(1)$ gauge field and Dirac (matter) fields) [35,36] have been shown to provide a set of field theoretical models for the Hodge theory where the local, covariant and continuous symmetry transformations (and their generators) correspond to the three de Rham cohomological operators $(d, \delta, \Delta)$ of differential geometry. A discrete symmetry has been shown to exist for all the above theories which corresponds to the Hodge duality $*$ operation of the differential geometry. Furthermore, the 2D free Abelian and self-interacting non-Abelian gauge theories have been shown to be topological $\ddagger$ in nature because of the existence of the topological invariants (as well as their proper recursion relations) and the appearance of the Lagrangian

\footnotetext{
${ }^{\dagger}$ The antisymmetric gauge potential $B_{\mu \nu}$ is defined through the 2-form $B=\frac{1}{2 !} B_{\mu \nu} d x^{\mu} \wedge d x^{\nu}$. The curvature term $H_{\mu \nu \kappa}$ is defined through the application of $d$ on $B$ (i.e. $H=d B, H=\frac{1}{3 !} H_{\mu \nu \kappa} d x^{\mu} \wedge d x^{\nu} \wedge d x^{\kappa}$ ). The gauge-fixing term $\left(\partial_{\mu} B^{\mu \nu}\right)$, on the other hand, is defined through the application of $\delta$ on $B$ (i.e. $\delta B=-* d * B=\partial_{\mu} B^{\mu \nu} d x_{\nu}$ ). Thus, in some sense, the gauge-fixing term is 'Hodge dual' to the curvature term because $d$ and $\delta=-* d *$ are Hodge dual to each-other (see, e.g., [26-30] for details).

${ }^{\ddagger}$ A (gauge) theory defined on the flat spacetime manifold (with a flat spacetime metric) and having no propagating degrees of freedom associated with the basic (gauge) field of the theory (see, e.g., [37]).
} 
density and symmetric energy momentum tensor which could be expressed as the sum of the (anti-)BRST and (anti-)co-BRST anti-commutators [33,38-41]. The geometrical interpretations for all the symmetry transformations and corresponding conserved charges (as generators) have been discussed in the framework of superfield formulation (see, e.g., [38-43] for details). In a recent set of papers [39-43], for the first time, the geometrical origin for the topological and cohomological aspects of these theories have been provided in the framework of superfield formulation. Thus, it will be noted that the above cited theories have very rich mathematical structure associated with them as they belong to a new class of topological field theories (TFTs) where the appearance of the Lagrangian density (and the symmetric energy momentum tensor) is like the Witten type TFTs [44] but the symmetries of these theories are just like Schwarz type (without a topological shift symmetry which is a characteristic feature of the existence of a Witten type TFT) [45].

It is obvious from the above discussions that the 4D 2-form Abelian gauge theory and 2D free Abelian and self-interacting non-Abelian gauge theories are prototype examples of a class of field theoretical models for the Hodge theory. The latter 2D theories are also exact TFTs because, the existence of (anti-)BRST and (anti-)co-BRST symmetries in the theory, turns out to be responsible for gauging out both the propagating degrees of freedom of 2D photon (and/or 2D gluon) (see, e.g., [31-33,37] for details). In the recent past, there has been some interest in studying almost TFTs (or quasi-TFTs) which involve constraints that leave out only a finite number of degrees of freedom in the theory $[37,46]$. So far, the (quasi-)topological and cohomological nature of the 4D 2-form gauge theory have not been studied together in a complementary fashion. The purpose of the present paper is to study the 4D 2-form Abelian gauge theory from the point of view of gaining some insights into the theoretical structure behind its quasi-topological as well as cohomological nature. We are propelled to go for such studies because of the close kinship that exists between this theory and the 2D free (and self-interacting) (non-)Abelian gauge theories. Our study is essential mainly on four counts: (i) any new property associated with the 4D Abelain 2-form gauge theory will turn out to be useful in the context of modern developments in (super)string theories and related topological and/or solitonic extended objects. (ii) The present theory is the first example of a $(3+1)$-dimensional gauge theory that provides a field theoretical model for the Hodge theory. It would be interesting, from the cohomological as well as topological points of view, to study this theory in more detail so that the idea of HDT and its connection with the (quasi-)TFTs can be put on a firmer footing. Specially, one should look for the quasi-topological nature of this theory as there is already a scalar degree of freedom associated with $B_{\mu \nu}$ in $4 \mathrm{D}$. (iii) The study of this theory on 4D manifold with flat spacetime metric is the first step in the direction to our main goal of studying this theory on the curved spacetime manifold with a non-trivial spacetime dependent metric. (iv) Our present study might turn out to be useful in the study of an "interacting" theory where $B \wedge F$ term is present. It might also give some insights into the way to attempt the study of non-Abelian 2-form gauge theory in the "extended" framework of BRST formulation. 
In our present paper, we shall concentrate mainly on the (anti-)BRST and (anti-)coBRST invariant versions ( $\mathrm{cf}(2.1)$ and $(2.8)$ below) of the following $(3+1)$-dimensional ${ }^{\S}$ free Kalb-Ramond Lagrangian density $[1,2,8]$

$$
\mathcal{L}_{0}=\frac{1}{12} H^{\mu \nu \kappa} H_{\mu \nu \kappa} \equiv \frac{1}{2} \mathcal{B}_{\mu} \mathcal{B}^{\mu}-\frac{1}{3 !} \varepsilon_{\mu \nu \kappa \zeta} \mathcal{B}^{\mu} H^{\nu \kappa \zeta}
$$

where $H_{\mu \nu \kappa}=\partial_{\mu} B_{\nu \kappa}+\partial_{\nu} B_{\kappa \mu}+\partial_{\kappa} B_{\mu \nu}$ is the curvature tensor constructed from the antisymmetric tensor field $B_{\mu \nu}$ and the vector auxiliary field $\mathcal{B}_{\mu}$ has been introduced to linearize the quadratic kinetic energy term $\left(\frac{1}{12} H^{\mu \nu \kappa} H_{\mu \nu \kappa}\right)$. It is evident that, in the above, $\mathcal{B}_{\mu}$ and $H_{\mu \nu \kappa}$ have the following inter-relationships due to the Euler-Lagrange equation of motion

$$
\mathcal{B}_{\mu}=\frac{1}{3 !} \varepsilon_{\mu \nu \kappa \zeta} H^{\nu \kappa \zeta} \equiv \frac{1}{2} \varepsilon_{\mu \nu \kappa \zeta} \partial^{\nu} B^{\kappa \zeta} \quad H_{\mu \nu \kappa}=\varepsilon_{\mu \nu \kappa \zeta} \mathcal{B}^{\zeta} .
$$

We shall demonstrate, in our present endeavour, that (i) the off-shell nilpotent (anti-)BRST and (anti-)co-BRST invariant Lagrangian density (2.1) can be expressed as the sum of: (a) the kinetic energy term and an (anti-)BRST exact part and/or (b) the gauge-fixing term and an (anti-)co-BRST exact part. This feature is similar to the 2D free Abelian and self-interacting non-Abelian gauge theories which are topological in nature [33,39,40]. (ii) There exist twelve sets of (anti-)BRST and (anti-)co-BRST invariant quantities for the Lagrangian density (2.1) that obey the recursion relations that are reminiscent of such relationships in the context of exact TFTs. (iii) The Lagrangian density (2.8) could be expressed, in terms of the on-shell nilpotent (anti-)BRST and (anti-)co-BRST charges, as the sum of (anti-)BRST and (anti-)co-BRST anti-commutators but for a factor of half (cf eqn (4.14) below). (iv) The 4D free Abelian 2-form gauge theory is very likely a candidate for the field theoretical model for a q-TFT and, of course, it is a very beautiful as well as an exact field theoretical model for the Hodge theory [25].

The outline of our paper is as follows. In section 2, we set up the notations as well as conventions by recapitulating some of the salient features of our earlier work [25] on the existence of off-shell nilpotent (anti-)BRST and (anti-)co-BRST symmetries in the Lagrangian formulation. We also discuss briefly the on-shell nilpotent version of these symmetries for our later convenience. Section 3 is central of our present paper and is devoted to the discussion of some special features associated with the 4D 2-form Abelian gauge theory. These features are mainly (i) the proof of this theory to be a field theoretical model for the Hodge theory [25], and (ii) its quasi-topological nature. Both these issues are so elegantly intertwined with each-other that first we present some of the key cohomological properties [25] and, then only, we discuss the quasi-topological nature. We compare and contrast some of the decisive properties of the 4D 2-form and 2D one-form Abelian gauge theories

\footnotetext{
${ }^{\S}$ We adopt here the conventions and notations in which the $4 \mathrm{D}$ flat Minkowski metric is: $\eta_{\mu \nu}=$ diag $(+1,-1,-1,-1)$ and totally anti-symmetric Levi-Civita tensor $\left(\varepsilon_{\mu \nu \kappa \zeta}\right)$ is chosen such that: $\varepsilon_{0123}=$ $+1=-\varepsilon^{0123}, \varepsilon_{0 i j k}=\varepsilon_{i j k}=-\varepsilon^{i j k}, \varepsilon_{\mu \nu \kappa \zeta} \varepsilon^{\mu \nu \kappa \zeta}=-4 !, \varepsilon_{\mu \nu \kappa \zeta} \varepsilon^{\mu \nu \kappa \eta}=-3 ! \delta_{\zeta}^{\eta}, \varepsilon_{\mu \nu \kappa \zeta} \varepsilon^{\mu \nu \eta \sigma}=-2 !\left(\delta_{\kappa}^{\eta} \delta_{\zeta}^{\sigma}-\delta_{\zeta}^{\eta} \delta_{\kappa}^{\sigma}\right)$ etc. Here Greek indices: $\mu, \nu, \kappa . .=0,1,2,3$ stand for the spacetime directions on the manifold and Latin indices: $i, j, k \ldots=1,2,3$ correspond to the space directions only.
} 
in section 4. Finally, in section 5, we make some concluding remarks.

\section{Preliminary: (co-)BRST symmetries}

We briefly recapitulate here the off-shell- as well as on-shell nilpotent (anti-)BRST and (anti-)dual-BRST symmetries which would be useful for our later discussions.

\subsection{Off-shell nilpotent symmetries}

We provide here a concise synopsis of our earlier work [25] on the existence of the offshell nilpotent $\left(s_{(A) B}^{2}=s_{(A) D}^{2}=0\right)$ (anti-)BRST $\left(s_{(A) B}\right)$ and (anti-)dual-BRST $\left(s_{(A) D}\right)$ symmetries that are respected together by the following linearized version of the Lagrangian density for the 2-form free Abelian gauge theory in $(3+1)$-dimensions [25]

$$
\begin{aligned}
\mathcal{L}_{B}^{(l)} & =\frac{1}{2} \mathcal{B}^{\mu} \mathcal{B}_{\mu}-\mathcal{B}^{\mu}\left(\frac{1}{2} \varepsilon_{\mu \nu \kappa \zeta} \partial^{\nu} B^{\kappa \zeta}-\partial_{\mu} \phi_{2}\right)+B^{\mu}\left(\partial^{\nu} B_{\nu \mu}-\partial_{\mu} \phi_{1}\right)-\frac{1}{2} B^{\mu} B_{\mu} \\
& -\partial_{\mu} \bar{\beta} \partial^{\mu} \beta+\left(\partial_{\mu} \bar{C}_{\nu}-\partial_{\nu} \bar{C}_{\mu}\right)\left(\partial^{\mu} C^{\nu}\right)+\rho\left(\partial_{\mu} C^{\mu}+\lambda\right)+\left(\partial_{\mu} \bar{C}^{\mu}+\rho\right) \lambda
\end{aligned}
$$

where $B_{\mu}, \mathcal{B}_{\mu}, \phi_{1}, \phi_{2}$ are the bosonic auxiliary fields and $B_{\mu \nu}$ is the bosonic antisymmetric tensor field (all with ghost number equal to zero), the scalar fields $\rho$ and $\lambda$ are the fermionic (i.e. $\rho^{2}=\lambda^{2}=0, \rho \lambda+\lambda \rho=0$ ) auxiliary (ghost) fields (with ghost number equal to $\mp 1$ ), the vector fermionic fields $\left(\bar{C}_{\mu}\right) C_{\mu}$ (i.e. $\left(C_{\mu}\right)^{2}=\left(\bar{C}_{\mu}\right)^{2}=0, C_{\mu} C_{\nu}+C_{\nu} C_{\mu}=0, C_{\mu} \bar{C}_{\nu}+\bar{C}_{\nu} C_{\mu}=$ $0, \bar{C}_{\mu} \bar{C}_{\nu}+\bar{C}_{\nu} \bar{C}_{\mu}=0$, etc.) are the (anti-)ghost fields (with ghost number $\mp 1$ ) and bosonic fields $(\bar{\beta}) \beta$ are the bosonic (anti-)ghost fields (with ghost number $\mp 2$ ). In fact, the above Lagrangian density remains quasi-invariant

$$
\begin{aligned}
& s_{B} \mathcal{L}_{B}^{(l)}=\partial_{\mu}\left[B^{\mu} \lambda-\rho \partial^{\mu} \beta+B_{\nu}\left(\partial^{\mu} C^{\nu}-\partial^{\mu} C^{\nu}\right)\right] \\
& s_{D} \mathcal{L}_{B}^{(l)}=-\partial_{\mu}\left[\lambda \partial^{\mu} \bar{\beta}+\rho \mathcal{B}^{\mu}+\mathcal{B}_{\nu}\left(\partial^{\mu} \bar{C}^{\nu}-\partial^{\nu} \bar{C}^{\mu}\right)\right]
\end{aligned}
$$

under the following off-shell nilpotent $\left(s_{B}^{2}=0\right) \operatorname{BRST}\left(s_{B}\right)$

$$
\begin{array}{lccc}
s_{B} B_{\mu \nu}=\left(\partial_{\mu} C_{\nu}-\partial_{\nu} C_{\mu}\right) & s_{B} C_{\mu}=\partial_{\mu} \beta & s_{B} \beta=0 & s_{B} \bar{C}_{\mu}=B_{\mu} \\
s_{B} B_{\mu}=0 & s_{B} \phi_{1}=-\lambda & s_{B} \lambda=0 & s_{B} \bar{\beta}=\rho \\
s_{B} \rho=0 & s_{B} \mathcal{B}_{\mu}=0 & s_{B} \phi_{2}=0 & s_{B}\left(\partial_{\mu} B_{\nu \kappa}\right)=0
\end{array}
$$

and the off-shell nilpotent $\left(s_{D}^{2}=0\right)$ dual(co)-BRST $\left(s_{D}\right)$ transformations $~ ฯ$

$$
\begin{array}{lccc}
s_{D} B_{\mu \nu}=\varepsilon_{\mu \nu \kappa \zeta} \partial^{\kappa} \bar{C}^{\zeta} & s_{D} \bar{C}_{\mu}=-\partial_{\mu} \bar{\beta} & s_{D} \bar{\beta}=0 & s_{D} C_{\mu}=\mathcal{B}_{\mu} \\
s_{D} \mathcal{B}_{\mu}=0 & s_{D} \phi_{2}=-\rho & s_{D} \rho=0 & s_{D} \beta=\lambda \\
s_{D} \lambda=0 & s_{D} B_{\mu}=0 & s_{D} \phi_{1}=0 & s_{D}\left(\partial^{\mu} B_{\mu \nu}\right)=0 .
\end{array}
$$

IWe adopt here the conventions and notations of [47] for the (co-)BRST transformations. In fact, in its totality, a nilpotent $\left(\delta_{(D) B}^{2}=0\right)\left(\right.$ co-)BRST transformation $\delta_{(D) B}$ is equal to the product of an anti-commuting number $\eta$ and the transformations $s_{(D) B}$ as: $\delta_{(D) B}=\eta s_{(D) B}$ with $s_{(D) B}^{2}=0$. 
It can be readily checked that under the following discrete symmetry transformations on the bosonic as well as fermionic fields of the theory

$$
\begin{array}{lcc}
\phi_{1} \rightarrow \pm i \phi_{2} & \phi_{2} \rightarrow \mp i \phi_{1} & B_{\mu} \rightarrow \pm i \mathcal{B}_{\mu} \\
\mathcal{B}_{\mu} \rightarrow \mp i B_{\mu} & B_{\mu \nu} \rightarrow \mp \frac{i}{2} \varepsilon_{\mu \nu \kappa \zeta} B^{\kappa \zeta} \\
C_{\mu} \rightarrow \pm i \bar{C}_{\mu} & \beta \rightarrow \mp i \bar{\beta} & \bar{\beta} \rightarrow \pm i \beta \\
\rho \rightarrow \pm i \lambda & \lambda \rightarrow \pm i \rho & \bar{C}_{\mu} \rightarrow \pm i C_{\mu}
\end{array}
$$

the above Lagrangian density (2.1) remains invariant. At this juncture, some of the salient points of our discussions, are (i) the ghost part of the Lagrangian density $\mathcal{L}_{g}=-\partial_{\mu} \bar{\beta} \partial^{\mu} \beta+$ $\left(\partial_{\mu} \bar{C}_{\nu}-\partial_{\nu} \bar{C}_{\mu}\right)\left(\partial^{\mu} C^{\nu}\right)+\rho\left(\partial_{\mu} C^{\mu}+\lambda\right)+\left(\partial_{\mu} \bar{C}^{\mu}+\rho\right) \lambda$ remains invariant in itself under (2.4) but the kinetic energy term and the gauge-fixing term exchange with each-other under the same transformations (i.e. eqn (2.4)). Exploiting this observation, one can derive the off-shell nilpotent $\left(s_{A B}^{2}=s_{A D}^{2}=0\right)$ anti-BRST $\left(s_{A B}\right)$ and anti-co-BRST $\left(s_{A D}\right)$ symmetries from (2.3) by the substitution $C_{\mu} \rightarrow \pm i \bar{C}_{\mu}, \bar{C}_{\mu} \rightarrow \pm i C_{\mu}, \beta \rightarrow \mp i \bar{\beta}, \bar{\beta} \rightarrow \pm i \beta, \rho \rightarrow \pm i \lambda, \lambda \rightarrow \pm i \rho$. (ii) Under the (anti-)BRST and (anti-)co-BRST symmetry transformations, it is the kinetic energy term (more precisely $H=d B$ ) and the gauge-fixing term (i.e. $\partial^{\kappa} B_{\kappa \mu} d x^{\mu}=\delta B, \delta=$ $\pm * d *$ ) that are found to remain invariant, respectively. (iii) The anticommutator of the (anti-)BRST and (anti-)co-BRST symmetries (i.e. $\left\{s_{B}, s_{D}\right\}=\left\{s_{A B}, s_{A D}\right\}=s_{W}$ ) leads to the definition of a bosonic symmetry transformation $\left(s_{W}\right)$ as (see, e.g., [31-33])

$$
\begin{array}{lc}
s_{W} B_{\mu \nu}=\partial_{\mu} \mathcal{B}_{\nu}-\partial_{\nu} \mathcal{B}_{\mu}+\varepsilon_{\mu \nu \kappa \xi} \partial^{\kappa} B^{\xi} & s_{W} C_{\mu}=\partial_{\mu} \lambda \\
s_{W}\left(\phi_{1}, \phi_{2}, B_{\mu}, \mathcal{B}_{\mu}, \rho, \lambda, \beta, \bar{\beta}\right)=0 & s_{W} \bar{C}_{\mu}=-\partial_{\mu} \rho \\
s_{W} \mathcal{L}_{B}=\partial_{\mu}\left[\rho \partial^{\mu} \lambda-\left(\partial^{\mu} \rho\right) \lambda+B^{\kappa} \partial^{\mu} \mathcal{B}_{\kappa}-\mathcal{B}^{\kappa} \partial^{\mu} B_{\kappa}+\mathcal{B}^{\mu}\left(\partial_{\kappa} B^{\kappa}\right)-B^{\mu}\left(\partial_{\kappa} \mathcal{B}^{\kappa}\right)\right]
\end{array}
$$

Under the above transformations, the ghost fields either do not transform or transform to a vector gauge transformation (i.e. $s_{W} C_{\mu}=\partial_{\mu} \lambda, s_{W} \bar{C}_{\mu}=-\partial_{\mu} \rho$ ). (iv) In addition to the above transformations, there is a global scale symmetry transformation (with global parameter $\Sigma$ ) which corresponds to the ghost symmetry $s_{g}$. The infinitesimal version of this symmetry is

$$
\begin{array}{lcc}
s_{g} B_{\mu \nu}=s_{g} B_{\mu}=s_{g} \mathcal{B}_{\mu}=s_{g} \phi_{1}=s_{g} \phi_{2}=0 \\
s_{g} C_{\mu}=\Sigma C_{\mu} & s_{g} \bar{C}_{\mu}=-\Sigma \bar{C}_{\mu} & s_{g} \rho=-\Sigma \rho \\
s_{g} \lambda=\Sigma \lambda & s_{g} \bar{\beta}=-2 \Sigma \bar{\beta} & s_{g} \beta=+2 \Sigma \beta .
\end{array}
$$

Thus, there are six symmetries in the theory which are local, covariant as well as continuous and four (i.e. $s_{(A) B}^{2}=0, s_{(A) D}^{2}=0$ ) of them are nilpotent of order two.

\subsection{On-shell nilpotent symmetries}

It is essential for our later discussions to elaborate on the presence of on-shell nilpotent $\left(s_{(a) b}^{2}=s_{(a) d}^{2}=0\right)\left(\right.$ anti-)BRST $\left(s_{(a) b}\right)$ and (anti-)co-BRST $\left(s_{(a) d}\right)$ symmetries in the theory. For this purpose, the following equations of motion, derived from (2.1)

$$
\begin{aligned}
& B_{\mu}=\left(\partial^{\sigma} B_{\sigma \mu}-\partial_{\mu} \phi_{1}\right) \quad \lambda=-\frac{1}{2}(\partial \cdot C) \equiv-\frac{1}{2}\left(\partial_{\mu} C^{\mu}\right) \\
& \mathcal{B}_{\mu}=\left(\frac{1}{2} \varepsilon_{\mu \nu \kappa \zeta} \partial^{\nu} B^{\kappa \zeta}-\partial_{\mu} \phi_{2}\right) \quad \rho=-\frac{1}{2}(\partial \cdot \bar{C}) \equiv-\frac{1}{2}\left(\partial_{\mu} \bar{C}^{\mu}\right)
\end{aligned}
$$


play a very decisive role. In fact, it can be readily checked that, with the help of (2.7), the Lagrangian density (2.1) can be recast as

$$
\begin{aligned}
\mathcal{L}_{b}^{(0)} & =\frac{1}{2}\left(\partial^{\sigma} B_{\sigma \mu}-\partial_{\mu} \phi_{1}\right)\left(\partial_{\kappa} B^{\kappa \mu}-\partial^{\mu} \phi_{1}\right) \\
& -\frac{1}{2}\left(\frac{1}{2} \varepsilon_{\mu \nu \kappa \zeta} \partial^{\nu} B^{\kappa \zeta}-\partial_{\mu} \phi_{2}\right)\left(\frac{1}{2} \varepsilon^{\mu \xi \sigma \eta} \partial_{\xi} B_{\sigma \eta}-\partial^{\mu} \phi_{2}\right) \\
& -\partial_{\mu} \beta \partial^{\mu} \beta+\left(\partial_{\mu} \bar{C}_{\nu}-\partial_{\nu} \bar{C}_{\mu}\right)\left(\partial^{\mu} C^{\nu}\right)-\frac{1}{2}(\partial \cdot \bar{C})(\partial \cdot C) .
\end{aligned}
$$

The equations of motion that emerge from the above Lagrangian density are

$$
\square B_{\mu \nu}=\square \phi_{1}=\square \phi_{2}=\square \beta=\square \bar{\beta}=0 \quad \square C_{\mu}=\frac{3}{2} \partial_{\mu}(\partial \cdot C) \quad \square \bar{C}_{\mu}=\frac{3}{2} \partial_{\mu}(\partial \cdot \bar{C}) .
$$

The Lagrangian density (2.8) is endowed with the following on-shell nilpotent $\left(s_{b}^{2} \phi_{1} \sim\right.$ $\square \beta=0, s_{b}^{2} \bar{\beta} \sim \square \phi_{1}=0, s_{b}^{2} \bar{C}_{\mu} \sim \square C_{\mu}-\frac{3}{2} \partial_{\mu}(\partial \cdot C)=0$ ) BRST symmetry transformations

$$
\begin{array}{lccc}
s_{b} B_{\mu \nu}=\left(\partial_{\mu} C_{\nu}-\partial_{\nu} C_{\mu}\right) & s_{b} C_{\mu}=\partial_{\mu} \beta & s_{b} \beta=0 & s_{b} \bar{C}_{\mu}=\left(\partial^{\nu} B_{\nu \mu}-\partial_{\mu} \phi_{1}\right) \\
s_{b} \phi_{1}=\frac{1}{2}(\partial \cdot C) \quad s_{b} \bar{\beta}=-\frac{1}{2}(\partial \cdot \bar{C}) & s_{b} \phi_{2}=0 & s_{b}\left(\partial_{\mu} B_{\nu \kappa}\right)=0
\end{array}
$$

together with the on-shell nilpotent $\left(s_{d}^{2} \phi_{2} \sim \square \bar{\beta}=0, s_{b}^{2} \beta \sim \square \phi_{2}=0, s_{b}^{2} C_{\mu} \sim \square \bar{C}_{\mu}-\right.$ $\left.\frac{3}{2} \partial_{\mu}(\partial \cdot \bar{C})=0\right)$ dual(co-)BRST transformations as listed below

$$
\begin{aligned}
& s_{d} B_{\mu \nu}=\varepsilon_{\mu \nu \kappa \zeta} \partial^{\kappa} \bar{C}^{\zeta} \quad s_{d} \bar{C}_{\mu}=-\partial_{\mu} \bar{\beta} \quad s_{d} C_{\mu}=\left(\frac{1}{2} \varepsilon_{\mu \nu \kappa \sigma} \partial^{\nu} B^{\kappa \sigma}-\partial_{\mu} \phi_{2}\right) \\
& s_{d} \bar{\beta}=0 \quad s_{d} \phi_{2}=\frac{1}{2}(\partial \cdot \bar{C}) \quad s_{d} \beta=-\frac{1}{2}(\partial \cdot C) \quad s_{d} \phi_{1}=0 \quad s_{d}\left(\partial^{\kappa} B_{\kappa \mu}\right)=0 .
\end{aligned}
$$

Exploiting the discrete symmetry transformations of the ghost fields in (2.4), it is straightforward to obtain the on-shell nilpotent anti-BRST $\left(s_{a b}\right)$ and anti-co-BRST $\left(s_{a d}\right)$ transformations from (2.10) and (2.11) respectively. Similarly, the on-shell version of the bosonic symmetry $\left(s_{w}\right)$ transformations $(2.5)$ can be obtained by substituting for the auxiliary fields from equation (2.7). The ghost symmetry transformations on the ghost fields in (2.6) remain intact (modulo the scale transformations on the auxiliary fields $B_{\mu}, \mathcal{B}_{\mu}, \rho, \lambda$ which no longer exist in the theory due to the Euler-Lagrange equations of motion (2.7)).

\section{Special features}

We discuss here some of the distinguished properties of the 4D free Abelian 2-form gauge theory keeping in mind similar features in the context of one-form gauge theories.

\subsection{Two-form gauge theory as the Hodge theory}

For the present paper to be self-contained, we briefly mention some of the key results of our earlier work [25] in a somewhat different manner than what has been discussed there. These results are intimately connected with the proof of the fact that the 4D 2-form gauge theory is a field theoretic model for the Hodge theory. In this work [25], all the de Rham cohomology operators $(d, \delta, \Delta)$ are identified with some specific symmetry transformations (and their generators) for the Lagrangian density of the theory. It is straightforward to 
check that all the six symmetries (on-shell as well as off-shell versions) obey the following operator form of the algebra $\|$

$$
\begin{aligned}
& s_{(a) b}^{2}=0 \quad s_{(a) d}^{2}=0 \quad\left[s_{w}, s_{r}\right]=0 \quad r=b, a b, d, a d, g \\
& \left\{s_{b}, s_{a b}\right\}=0 \quad\left\{s_{d}, s_{a d}\right\}=0 \quad i\left[s_{g}, s_{b(a d)}\right]=+s_{b(a d)} \\
& i\left[s_{g}, s_{d(a b)}\right]=-s_{b(a d)} \quad\left\{s_{b}, s_{d}\right\}=s_{w}=\left\{s_{a b}, s_{a d}\right\}
\end{aligned}
$$

which is reminiscent of the algebraic structure obeyed by the cohomological operators $(d, \delta, \Delta)$. A noteworthy point is the fact that the discrete symmetry transformations (2.4) correspond to the Hodge duality * operation of the differential geometry on a compact manifold. In this context, it can be checked that the following relationships between the (anti-)co-BRST and (anti-)BRST transformations are satisfied:

$$
s_{(d, D)} \Phi= \pm * s_{(b, B)} * \Phi \quad s_{(a d, A D)} \Phi= \pm * s_{(a b, A B)} * \Phi
$$

where $\Phi$ stands for the generic field of the Lagrangian densities (2.1) and (2.8). In the above, the \pm signs for a particular field are dictated by the corresponding signs that appear when we take two successive $*$ operation on that field [48-50]. In more general terms, this statement can be expressed succinctly, for the generic field $\Phi$ of the theory, as

$$
*(* \Phi)= \pm \Phi
$$

where, as is evident, the $*$ operation is nothing but the discrete symmetry transformations in (2.4) for the fields. For the theory under consideration, it turns out that

$$
\begin{array}{ll}
*(* B)=+B & B=\beta, \bar{\beta}, \phi_{1}, \phi_{2}, B_{\mu \nu}, B_{\mu}, \mathcal{B}_{\mu} \\
*(* F)=-F & F=\rho, \lambda, C_{\mu}, \bar{C}_{\mu} .
\end{array}
$$

Thus, the $( \pm)$ signs in (3.2) find their physical interpretation in the sense that the nilpotent (anti-)co-BRST and (anti-)BRST transformations on the bosonic(fermionic) fields $(B) F$ of the 2-form gauge theory are related through the interplay between discrete and continuous symmetries of the theory. In explicit form, the above relationship can be expressed as: $s_{(d, D)} B=+* s_{(b, B)} * B, s_{(d, D)} F=-* s_{(b, B)} * F$ where $B$ and $F$ of the theory are defined in (3.4). As is self evident, a similar kind of relationship exists for the anti-BRST and anti-co-BRST transformations if we could exploit (2.4) judiciously.

The relationship in (3.2) is exactly same as the connection between cohomological operators $\delta$ and $d$ (i.e. $\delta= \pm * d *$ ). Whereas in differential geometry, the $( \pm)$ signs in $\delta= \pm * d *$ are dictated by the dimensionality ${ }^{* *}$ of the manifold on which the Hodge duality

\footnotetext{
"We have taken here the notations of the on-shell version of the symmetry transformations but the present algebraic structure is valid for the off-shell version of the symmetry transformations as well. Furthermore, a similar kind of operator algebra is obeyed by the off-shell as well as on-shell version of the generators (cf (5.1) below) which are nothing but the conserved Noether charges. It will be noted that (5.1), although written for the off-shell version, is also true for the on-shell version of the generators.

**In any arbitrary even $D$-dimensional manifold, $d$ and $\delta$ are related by: $\delta=-* d *$. In general, an inner product of a n-form in $D$ dimensional manifold leads to the relationship between $d$ and $\delta$ as: $\delta=(-1)^{D n+D+1} * d *$. Thus, for the odd dimensional manifold, we have $\delta=(-1)^{n} * d *($ see, e.g., [26,27]).
} 
is defined (see, e.g., [26-30]), it is evident that, in the context of 2-form gauge theory these signs are dictated by (3.3). Ultimately, it so happens that the signs in (3.3) are guided by the nature of field(s) of the theory under consideration. Other useful properties of the 2-form Abelian gauge theory, under the $*$ operation (corresponding to the discrete symmetry transformation (2.4)), are: (i) the Lagrangian density (2.1) remains invariant. The on-shell version of the (2.4) can be obtained by substituting for the auxiliary fields of the theory from (2.7). Under the latter transformations, the Lagrangian density (2.8) remains invariant. (ii) The continuous symmetry transformations (on-shell as well as off-shell versions) and corresponding symmetry generators $Q_{r}, r=B(b), A B(a b), D(d), A D(a d), g$ of the theory transform, under (2.4), as

$$
\begin{array}{lccc}
s_{(A) B} \rightarrow s_{(A) D} & s_{(A) D} \rightarrow-s_{(A) B} \quad s_{(W, w)} \rightarrow-s_{(W, w)} \quad s_{g} \rightarrow-s_{g} \\
Q_{(A) B} \rightarrow Q_{(A) D} \quad Q_{(A) D} \rightarrow-Q_{(A) B} \quad Q_{(W, w)} \rightarrow-Q_{(W, w)} \quad Q_{g} \rightarrow-Q_{g} \\
s_{(a) b} \rightarrow s_{(a) d} \quad s_{(a) d} \rightarrow-s_{(a) b} \quad Q_{(a) b} \rightarrow Q_{(a) d} & Q_{(a) d} \rightarrow-Q_{(a) b}
\end{array}
$$

where the local expressions for the conserved charges $Q_{r}$ can be found in [25]. For our present discussions, these expressions are not required to be quoted here. (iii) The above transformations are such that the algebraic structure of (3.1) does not change at all. (iv) There exists an inverse relationship vis-à-vis relations (3.2) as given below

$$
s_{(b, B)} \Phi=\mp * s_{(d, D)} * \Phi \quad s_{(a b, A B)} \Phi=\mp * s_{(a d, A D)} * \Phi
$$

where, it can be noted, the signs have changed in the r.h.s. In explicit form, the above transformations can be expressed as: $s_{(b, B)} B=-* s_{(d, D)} * B, s_{(b, B)} F=+* s_{(d, D)} * F$ where $B$ and $F$ are the bosonic and fermionic fields of the theory. This fact should be contrasted with the case of $2 \mathrm{D}$ free Abelian gauge theory, for which, the signs are same in the equations corresponding to (3.2) and (3.6) [33]. This peculiarity happens because the duality in 4D and 2D are different [48-50]. (v) The transformations $s_{(b, B)} \rightarrow s_{(d, D)}, s_{(d, D)} \rightarrow-s_{(b, B)}$ under the Hodge $*$ operation are reminiscent of the duality transformations for the electric $(\mathbf{E})$ and magnetic $(\mathbf{B})$ fields in the case of source free Maxwell equations where $\mathbf{E} \rightarrow \mathbf{B}, \mathbf{B} \rightarrow-\mathbf{E}$.

All the above properties establish the fact that the 4D free Abelian 2-form gauge theory is a field theoretical model for the Hodge theory because the local, covariant and continuous symmetry transformations (the off-shell as well as the on-shell versions) for the Lagrangian density of the theory (and the corresponding Noether conserved charges) obey an algebra that is reminiscent of the algebra obeyed by the de Rham cohomological operators $(d, \delta, \Delta)$. The discrete symmetry transformations (2.4) (and its on-shell version) are the analogue of the Hodge duality $*$ operation of the differential geometry. As far as the ghost number consideration of the states in the quantum Hilbert space are concerned, it turns out that the set $(d, \delta, \Delta)$ has a one-to-two mappings with the conserved charges (or generators) of the theory, namely; $d \rightarrow Q_{b(a d)}, \delta \rightarrow Q_{d(a b)}, \Delta \rightarrow Q_{w}=\left\{Q_{b}, Q_{d}\right\}=\left\{Q_{a b}, Q_{a d}\right\}$. The basic theoretical reason behind the existence of such a mapping can be explained in the framework of superfield formulation of the present theory as has been done for the one-form free Abelian 
(and self-interacting non-Abelian) gauge theory (see, e.g., [38-43] for details). Because of the existence of the above mapping, the analogue of the Hodge decomposition theorem can be defined in the quantum Hilbert space of states [25]. In fact, all the above cited properties of this section are common to the $2 \mathrm{D}$ one-form free Abelian (and self-interacting non-Abelian) as well as 4D free Abelian 2-form gauge theories [25,31-36] except for the sign difference in the inverse relationship (3.6). To be more precise, the analogues of (3.2) and (3.6) for the $2 \mathrm{D}$ theories bear the same signs on the r.h.s. in contrast to the $4 \mathrm{D}$ theories where there is a sign flip on the r.h.s. of the relationships (3.2) and (3.6).

\subsection{Towards topological aspects}

It can be checked explicitly that the linearized version of the the Lagrangian density (2.1) can be expressed in the following two different ways by exploiting the off-shell nilpotent $\left(s_{B}^{2}=s_{D}^{2}=0\right)\left(\right.$ co-)BRST symmetries $\left(s_{(D) B}\right)(\operatorname{cf}(2.3))$ of the theory

$$
\begin{gathered}
\mathcal{L}_{B}^{(l)}=B^{\mu}\left(\partial^{\sigma} B_{\sigma \mu}-\partial_{\mu} \phi_{1}\right)-\frac{1}{2} B^{\mu} B_{\mu}+s_{D}(G)+\partial_{\mu} Z^{\mu} \\
\mathcal{L}_{B}^{(l)}=\frac{1}{2} \mathcal{B}^{\mu} \mathcal{B}_{\mu}-\mathcal{B}^{\mu}\left(\frac{1}{2} \varepsilon_{\mu \nu \kappa \zeta} \partial^{\nu} B^{\kappa \zeta}-\partial_{\mu} \phi_{2}\right)+s_{B}(F)+\partial_{\mu} T^{\mu}
\end{gathered}
$$

where the expressions for $G, Z^{\mu}, F, T^{\mu}$, in terms of the local fields, are

$$
\begin{aligned}
G & =\frac{1}{2} C^{\mu}\left(\mathcal{B}_{\mu}-\varepsilon_{\mu \nu \kappa \zeta} \partial^{\nu} B^{\kappa \zeta}\right)-(\partial \cdot C+\lambda) \phi_{2}-(\partial \cdot \bar{C}+\rho) \beta \\
Z^{\mu} & =\mathcal{B}^{\mu} \phi_{2}-\beta \partial^{\mu} \bar{\beta}+\left(\partial^{\mu} \bar{C}^{\nu}-\partial^{\nu} \bar{C}^{\mu}\right) C_{\nu} \\
F & =\bar{C}^{\mu}\left(\partial^{\sigma} B_{\sigma \mu}-\frac{1}{2} B_{\mu}\right)+(\partial \cdot C+\lambda) \bar{\beta}+(\partial \cdot \bar{C}+\rho) \phi_{1} \\
T^{\mu} & =\bar{C}_{\nu}\left(\partial^{\mu} C^{\nu}-\partial^{\nu} C^{\mu}\right)-B^{\mu} \phi_{1}-\bar{\beta} \partial^{\mu} \beta .
\end{aligned}
$$

The above equation (3.7) can also be expressed in terms of the anti-BRST and anti-coBRST transformations by exploiting the discrete transformations (2.4) for the ghost fields. The analogue of expressions in (3.8), for the latter case, can also be computed by the substitution of transformations for the ghost fields in (2.4). Thus, it is evident that there are four ways to express the Lagrangian density (2.1) in terms of the four off-shell nilpotent symmetry transformations $\left(s_{(A) B}, s_{(A) D}\right)$ of the theory. In fact, this special feature of the 2 -form gauge theory is identical to the 2D free Abelian (and self-interacting non-Abelian) 1-form gauge theories which have been shown [33,39-41] to be topological in nature.

One of the interesting features of a field theory to be a topological field theory is the existence of topological invariants which obey a certain specific type of recursions relations (see, e.g., [37] for details). In fact, it has been shown for the one-form free 2D Abelian gauge theory (as well as the self-interacting 2D non-Abelian gauge theory) that there are four

sets of such invariants $\left(\tilde{I}_{k}\right) I_{k}$ and $\left(\tilde{J}_{k}\right) J_{k}$ w.r.t. the four conserved $\left(\dot{Q}_{(A) B}=\dot{Q}_{(A) D}=0\right)$ and nilpotent $\left(Q_{(A) B}^{2}=Q_{(A) D}^{2}=0\right)$ (anti-)BRST charges $Q_{(A) B}$ and (anti-)co-BRST charges $Q_{(A) D}$, namely;

$$
I_{k}=\oint_{C_{k}} V_{k} \quad \tilde{I}_{k}=\oint_{C_{k}} \tilde{V}_{k} \quad J_{k}=\oint_{C_{k}} W_{k} \quad \tilde{J}_{k}=\oint_{C_{k}} \tilde{W}_{k}
$$


where $\left(\tilde{V}_{k}\right) V_{k}$ and $\left(\tilde{W}_{k}\right) W_{k}$ are the k-forms $(k=0,1,2)$ and $C_{k}$ are the homology cycles on the 2D closed Riemann surface which is an Euclidean version of the 2D Minkowski manifold we started with. Euclideanization is essential to have meaning of the topological invariants, homology cycles etc. in the language of the curves in algebraic geometry as the Minkowski manifold is a non-compact manifold [51]. Since there is a single set of (anti-)ghost fields $(\bar{C}) C$ in one-form 2D (non-)Abelian gauge theories, we obtain first the two sets of invariants w.r.t. (co-)BRST charges. From these, the other two (w.r.t. anti-BRST and anti-co-BRST charges) can be computed by exploiting the discrete ghost symmetries of the theory [3134]. In contrast, in the present 2-form 4D Abelian gauge theory, there are three sets of (anti-)ghost fields, namely; $(\bar{\beta}) \beta,\left(\bar{C}_{\mu}\right) C_{\mu},(\rho) \lambda$ with ghost numbers: $(-2) 2,(-1) 1,(-1) 1$, respectively. Thus we, expect the existence of invariants including all these ghost fields. It can be checked that the following set (which starts with an invariant containing $\beta$ )

$$
\begin{aligned}
V_{0}(+2) & =(\partial \cdot B) \beta \\
V_{1}(+1) & =\left[\partial_{\mu}(\partial \cdot \bar{C}) \beta+(\partial \cdot B) C_{\mu}\right] d x^{\mu} \\
V_{2}(0) & =\left[\partial_{\mu}(\partial \cdot \bar{C}) C_{\nu}+\frac{1}{2}(\partial \cdot B) B_{\mu \nu}\right] d x^{\mu} \wedge d x^{\nu} \\
V_{3}(-1) & =\left[\frac{1}{2} \partial_{\mu}(\partial \cdot \bar{C}) B_{\nu \lambda}+\frac{1}{3 !}(\partial \cdot \bar{C}) H_{\mu \nu \kappa}\right] d x^{\mu} \wedge d x^{\nu} \wedge d x^{\kappa} \\
& \equiv d\left[\frac{1}{2}(\partial \cdot \bar{C}) B_{\nu \kappa} d x^{\nu} \wedge d x^{\kappa}\right] \\
V_{4} & =0 \quad\left(d^{2}=0\right) .
\end{aligned}
$$

is a set of invariants w.r.t. the conserved and nilpotent BRST charge $Q_{B}$. The noteworthy points, at this stage, are: (i) the zero-form $V_{0}$ is a BRST invariant quantity (i.e. $s_{B} V_{0}=0$ ). (ii) The three form $V_{3}$ turns out to be an exact form. Thus, we are unable to deduce the four-form $V_{4}$ for the theory on $4 \mathrm{D}$ manifold. (iii) The ghost numbers for the forms $V_{0}-V_{3}$ are $+2,+1,0,-1$ respectively. These numbers have been exploited to express uniquely the $k$-forms $V_{k}(n), k=0,1,2,3$ for $n=+2,+1,0,-1$. (iv) The above forms obey a recursion relation (a key characteristic feature of an exact TFT) as

$$
s_{B} I_{k}=d I_{k-1} \quad(k=1,2,3,4)
$$

where $d=d x^{\mu} \partial_{\mu}$ is the exterior derivative. (v) From the above k-forms $V_{k}$, one can compute easily the $k$-forms $\tilde{V}_{k}$ corresponding to the nilpotent anti-BRST charge $Q_{A B}$ by exploiting the discrete transformations for the ghost fields: $\beta \rightarrow \mp i \bar{\beta}, \bar{\beta} \rightarrow \pm i \beta, \rho \rightarrow \pm i \lambda, \lambda \rightarrow$ $\pm i \rho, C_{\mu} \rightarrow \pm i \bar{C}_{\mu}, \bar{C}_{\mu} \rightarrow \pm i C_{\mu}$. The resulting invariants $\tilde{I}_{k},(k=0,1,2,3,4)$ obey the same kind of recursion relations as (3.11) (i.e. $\left.s_{A B} \tilde{I}_{k}=d \tilde{I}_{k-1},(k=1,2,3,4)\right)$.

One can now compute the invariants w.r.t. the conserved and nilpotent co-BRST charge $Q_{D}$. The set $W_{k}(k=0,1,2,3,4)$, which constitutes the invariants $J_{k}$, is as follows

$$
\begin{aligned}
W_{0}(-2) & =(\partial \cdot \mathcal{B}) \bar{\beta} \\
W_{1}(-1) & =\left[\partial_{\mu}(\partial \cdot C) \bar{\beta}-(\partial \cdot \mathcal{B}) \bar{C}_{\mu}\right] d x^{\mu} \\
W_{2}(0) & =\left[-\partial_{\mu}(\partial \cdot C) \bar{C}_{\nu}+\frac{1}{4}(\partial \cdot \mathcal{B}) \varepsilon_{\mu \nu \kappa \sigma} B^{\kappa \sigma}\right] d x^{\mu} \wedge d x^{\nu} \\
W_{3}(+1) & =\frac{1}{4}\left[\partial_{\mu}(\partial \cdot C) B^{\kappa \sigma}+(\partial \cdot \bar{C}) \partial_{\mu} B^{\kappa \sigma}\right] \varepsilon_{\nu \eta \kappa \sigma} d x^{\mu} \wedge d x^{\nu} \wedge d x^{\eta} \\
& \equiv d\left[\frac{1}{4}(\partial \cdot \bar{C}) \varepsilon_{\mu \nu \kappa \sigma} B^{\kappa \sigma} d x^{\mu} \wedge d x^{\nu}\right] \\
W_{4} & =0 \quad\left(d^{2}=0\right) .
\end{aligned}
$$


Some of the salient features, at this juncture, are (i) from the above forms, one can compute the forms $\tilde{W}_{k}(k=0,1,2,3,4)$ of (3.9) by exploiting the discrete transformations on the bosonic as well as fermionic ghost fields of equation (2.4). (ii) The zero-forms $\tilde{W}_{0}(+2)$ and $W_{0}(-2)$ are invariant under the (anti-)co-BRST transformations $s_{(A) D}$ because $s_{A D} \tilde{W}_{0}(+2)=0, s_{D} W_{0}(-2)=0$. (iii) The ghost numbers for $J_{0}-J_{3}$, constructed from $W_{0}-W_{3}$, are $-2,-1,0,+1$ respectively. (iv) It is interesting to note that

$$
* V_{k}(n)=W_{k}(\tilde{n}) \quad * \tilde{V}_{k}(\tilde{n})=\tilde{W}_{k}(n) \quad \tilde{n}=-2, \mp 1,0 \quad n=+2, \pm 1,0
$$

where the $*$ operation corresponds to the discrete symmetry transformations in $(2.4)$. (v) The invariants $J_{k}, \tilde{J}_{k}$, constructed from $W_{k}(\tilde{n}), \tilde{W}_{k}(n)$, obey the following recursion relations

$$
s_{D} J_{k}=d J_{k-1} \quad s_{A D} \tilde{J}_{k}=d \tilde{J}_{k-1} \quad(k=1,2,3,4)
$$

which is the analogue of the similar relation in (3.11) w.r.t. the BRST charge $Q_{B}$.

Taking into account now the bosonic (anti-)ghost fields $(\bar{\beta}) \beta$ and the fermionic vector (anti-)ghost fields $\left(\bar{C}_{\mu}\right) C_{\mu}$, we can construct the following zero-forms $W_{0}$ and $V_{0}$

$$
W_{0}(-1)=-\left[\mathcal{B}_{\mu} \bar{C}^{\mu}+C^{\mu} \partial_{\mu} \bar{\beta}\right] \quad V_{0}(+1)=B_{\mu} C^{\mu}-\bar{C}^{\mu} \partial_{\mu} \beta
$$

that remain invariant under the (co-)BRST transformations $s_{(D) B}$ as $s_{D} W_{0}=0, s_{B} V_{0}=0$. In the above equation, the numbers in the round bracket (i.e. $( \pm 1)$ ) denote the ghost numbers for the zero-forms. The other non-trivial forms $V_{k}, k=1,2$, that are found to be consistent with the recursion relations (3.11) and can be constructed (with ghost numbers $0,-1)$ in terms of the (anti-)ghost fields $\left(\bar{C}_{\mu}\right) C_{\mu}$, are

$$
\begin{aligned}
V_{1}(0) & =\left[\left(\partial_{\kappa} \bar{C}_{\mu}\right) C^{\mu}+\bar{C}^{\mu} \partial_{\mu} C_{\kappa}+B^{\mu} B_{\kappa \mu}\right] d x^{\kappa} \\
V_{2}(-1) & =\left[\left(\partial_{\sigma} \bar{C}^{\mu}\right) B_{\kappa \mu}+\bar{C}^{\mu}\left(\partial_{\sigma} B_{\kappa \mu}\right)\right] d x^{\sigma} \wedge d x^{\kappa} \\
& \equiv d\left[\left(\bar{C}^{\mu} B_{\kappa \mu}\right) d x^{\kappa}\right] .
\end{aligned}
$$

It is evident that $V_{3}=V_{4}=0$ as $V_{2}(-1)$ turns out to be an exact form. Thus, recursion relations (cf (3.11)) allow the existence of the forms only up to degree two because, at order two itself, we obtain an exact form. It should be noted, at this stage, that there is another candidate $V_{1}^{(a)}(0)$ for degree one, namely;

$$
V_{1}^{(a)}(0)=\left[\left(\partial_{\kappa} \bar{C}_{\mu}\right) C^{\mu}+\bar{C}^{\mu}\left(\partial_{\kappa} C_{\mu}\right)\right] d x^{\kappa} \equiv d\left[\bar{C}_{\mu} C^{\mu}\right]
$$

which obeys the recursion relations (3.11). But we do not consider it because it is already an exact form (and, therefore, trivial). Now, consistent with the recursion relations (3.14), the higher order forms with respect to the co-BRST charge $Q_{D}$ are

$$
\begin{aligned}
W_{1}(0) & =-\left[\left(\partial_{\kappa} C_{\mu}\right) \bar{C}^{\mu}+C^{\mu}\left(\partial_{\mu} \bar{C}_{\kappa}\right)-\frac{1}{2} \varepsilon_{\kappa \mu \eta \zeta} \mathcal{B}^{\mu} B^{\eta \zeta}\right] d x^{\kappa} \\
W_{2}(-1) & =\frac{1}{2}\left[\left(\partial_{\sigma} C^{\mu}\right) B^{\eta \zeta}+C^{\mu} \partial_{\sigma} B^{\eta \zeta}\right] \varepsilon_{\kappa \mu \eta \zeta} d x^{\sigma} \wedge d x^{\kappa} \\
& \equiv \frac{1}{2} d\left[\varepsilon_{\kappa \mu \eta \zeta} C^{\mu} B^{\eta \zeta} d x^{\kappa}\right] .
\end{aligned}
$$


It is very much clear that $W_{3}=W_{4}=0$ because the 2 -form $W_{2}(-1)$ is itself an exact form. Thus, the series of invariants terminates here and $W_{3}$ and $W_{4}$ turn out to be trivially zero (because $d^{2}=0$ ). Analogous to $(3.17)$, there is an additional candidate $W_{1}^{(a)}(0)$ but it turns out to be an exact form (i.e. $W_{1}^{(a)}=-d\left[C_{\mu} \bar{C}^{\mu}\right]$ ). This is why, it has not been considered in the above series. It is obvious that the forms $\tilde{V}_{k}, \tilde{W}_{k}(k=0,1,2)$ with respect to the anti-BRST $\left(Q_{A B}\right)$ and anti-co-BRST $\left(Q_{A D}\right)$ charges can be computed from (3.15)-(3.18) by exploiting the discrete symmetry transformations on the ghost fields in (2.4). The ghost numbers for the invariants constructed from $\tilde{V}_{k}$ and $\tilde{W}_{k}$ would turn out to be $(-1,0,+1)$ and $(+1,0,-1)$ respectively and they would obey the recursion relations $s_{A B} \tilde{I}_{k}=d I_{k-1}, s_{A D} \tilde{J}_{k}=d \tilde{J}_{k-1},(k=1,2,3$,$) .$

Let us now concentrate on the invariants that are constructed with the ghost field $\lambda$ which carries the ghost number equal to +1 . The following set

$$
\begin{aligned}
& V_{0}(+1)=(\partial \cdot B) \lambda \\
& V_{1}(0) \quad=\left[\partial_{\mu}(\partial \cdot \bar{C}) \lambda+(\partial \cdot B) \partial_{\mu} \phi_{1}\right] d x^{\mu} \\
& V_{2}(-1)=-\left[\partial_{\mu}(\partial \cdot \bar{C}) \partial_{\nu} \phi_{1}\right] d x^{\mu} \wedge d x^{\nu} \equiv-d\left[(\partial \cdot \bar{C}) \partial_{\nu} \phi_{1} d x^{\nu}\right] \\
& V_{3}=V_{4}=0 \quad\left(d^{2}=0\right)
\end{aligned}
$$

obeys the recursion relations given in (3.11). From the BRST invariant $\left(s_{B} V_{0}(+1)=0\right)$ zero-form $V_{0}(+1)$ in the above, one can construct an additional set $V_{k}^{(a)}(k=1,2)$ as

$$
\begin{array}{ll}
V_{1}^{(a)}(0) & =\left[(\partial \cdot \bar{C}) \partial_{\mu} \lambda+\partial_{\mu}(\partial \cdot B) \phi_{1}\right] d x^{\mu} \\
V_{2}^{(a)}(-1) & =\left[\partial_{\mu}(\partial \cdot \bar{C}) \partial_{\nu} \phi_{1}\right] d x^{\mu} \wedge d x^{\nu} \equiv+d\left[(\partial \cdot \bar{C}) \partial_{\nu} \phi_{1} d x^{\nu}\right] \\
V_{3}^{(a)}=V_{4}^{(a)} & =0 \quad\left(d^{2}=0\right) .
\end{array}
$$

The above set also obeys the recursion relations of (3.11). It should be emphasized that there are two more candidates for the one-form $V_{1}(0)$ (with ghost number 0 ) which obey the recursion relations (3.11). But, they turn out to be trivial in the sense that they are found to be exact forms. The precise expressions for them are: $V_{1}^{(1)}(0)=d[(\partial \cdot \bar{C}) \lambda], V_{1}^{(2)}(0)=$ $-d\left[(\partial \cdot B) \phi_{1}\right]$. It is evident that, w.r.t. these trivial one-forms, there are no non-trivial 2 -forms in the recursion relations $(3.11)$ because $\left(d^{2}=0\right)$. Analogous to the above, a set of invariants can be constructed w.r.t. conserved $\left(\dot{Q}_{D}=0\right)$ and nilpotent $\left(Q_{D}^{2}=0\right)$ co-BRST charge $Q_{D}$. The corresponding forms $W_{k},(k=0,1,2,3,4)$, with self-evident notations, are

$$
\begin{aligned}
& W_{0}(-1)=(\partial \cdot \mathcal{B}) \rho \\
& W_{1}(0)=-\left[\partial_{\mu}(\partial \cdot C) \rho-(\partial \cdot \mathcal{B}) \partial_{\mu} \phi_{2}\right] d x^{\mu} \\
& W_{2}(+1)=\left[\partial_{\mu}(\partial \cdot C) \partial_{\nu} \phi_{2}\right] d x^{\mu} \wedge d x^{\nu} \equiv d\left[(\partial \cdot C) \partial_{\nu} \phi_{2} d x^{\nu}\right] \\
& W_{3}=W_{4}=0 \quad\left(d^{2}=0\right) \text {. }
\end{aligned}
$$

The invariants $J_{k}$ constructed from the above forms obey the recursion relations given in (3.14). There exist additional forms $W_{k}^{(a)}$ which can be constructed from the co-BRST invariant $\left(s_{D} W_{0}(-1)=0\right)$ zero-form $W_{0}(-1)$. These are as follows

$$
\begin{array}{ll}
W_{1}^{(a)}(0) & =-\left[(\partial \cdot C) \partial_{\mu} \rho-\partial_{\mu}(\partial \cdot \mathcal{B}) \phi_{2}\right] d x^{\mu} \\
W_{2}^{(a)}(+1) & =-\left[\partial_{\mu}(\partial \cdot C) \partial_{\nu} \phi_{2}\right] d x^{\mu} \wedge d x^{\nu} \equiv-d\left[(\partial \cdot C) \partial_{\nu} \phi_{2} d x^{\nu}\right] \\
W_{3}^{(a)}=W_{4}^{(a)} & =0 \quad\left(d^{2}=0\right) .
\end{array}
$$


The invariants constructed from the above also obey (3.14). There are a few comments in order now. (i) It is straightforward to check that the forms $\tilde{W}_{k}(k=0,1,2,3,4)$ corresponding to the conserved $\left(\dot{Q}_{A D}=0\right)$ and nilpotent $\left(Q_{A D}^{2}=0\right)$ anti-dual BRST charge $Q_{A D}$ can be derived from $W_{k}$ and $W_{k}^{(a)}$ by exploiting the discrete symmetry transformations on the ghost fields in (2.4). (ii) The invariants $\tilde{J}_{k}(k=0,1,2,3,4)$ constructed from these forms would obey the same kind of recursion relations as given in (3.14). (iii) It is interesting to note that if we exploit the full discrete transformations of the analogue of Hodge duality $*$ operation in (2.4), we obtain

$$
\begin{aligned}
& * V_{k}(n) \quad=W_{k}(\tilde{n}) \quad * \tilde{V}_{k}(\tilde{n})=\tilde{W}_{k}(n) \\
& * V_{k}^{(a)}(n)=W_{k}^{(a)}(\tilde{n}) \quad * \tilde{V}_{k}^{(a)}(\tilde{n})=\tilde{W}_{k}^{(a)}(n)
\end{aligned}
$$

where $n=+1,0,-1$ and $\tilde{n}=-1,0,+1$.

\section{One-form 2D- versus two-form 4D free Abelian gauge theories}

In this section, we shall compare and contrast between some of the key issues associated with $2 \mathrm{D}$ free vector Abelian gauge theory vis-à-vis 4D free Abelian antisymmetric gauge theory. For the sake of completeness and clarity, we first recapitulate some of the key properties of the $2 \mathrm{D}$ one-form Abelian gauge theory ${ }^{\dagger \dagger}$ [31-33]. Before we embark on addressing the difference(s) between these theories, it is important to emphasize that these theories have something in common. These key similarities are: (i) they are field theoretical models for the Hodge theory because the symmetries (and corresponding generators) of the Lagrangian density obey the same kind of algebra as obeyed by the de Rham cohomology operators $(d, \delta, \Delta)$. (ii) The analogue of the Hodge duality $*$ transformations in $(2.4)$ can be defined for the one-form gauge theory as well [33]. (iii) The (anti-)BRST and (anti-)co-BRST invariant quantities obey the same kind of recursion relations as that of a typical field theoretical examples of an exact TFT. (iv) These invariants terminate after reaching the differential-form that could be defined on the specific manifold with the maximum degree. Now, we concentrate on the discussion of a key difference between these theories. To this end in mind, we begin with the BRST invariant Lagrangian density for the free 2D Abelian gauge theory in the Feynman gauge (see, e.g. [20,47,52,53])

$$
\tilde{\mathcal{L}}_{b}=-\frac{1}{4} F_{\mu \nu} F^{\mu \nu}-\frac{1}{2}(\partial \cdot A)^{2}-i \partial_{\mu} \bar{C} \partial^{\mu} C \equiv \frac{1}{2} E^{2}-\frac{1}{2}(\partial \cdot A)^{2}-i \partial_{\mu} \bar{C} \partial^{\mu} C
$$

where only the electric field component $F_{01}=-\varepsilon^{\mu \nu} \partial_{\mu} A_{\nu}=E$ (with $\varepsilon_{01}=\varepsilon^{10}=+1$ ) of the curvature tensor $F_{\mu \nu}$ exists in $2 \mathrm{D}$ and $F_{\mu \nu}$ is derived from the 2-form $F=d A$ (with $d=$ $d x^{\mu} \partial_{\mu}, d^{2}=0$ and $\left.A=d x^{\mu} A_{\mu}\right)$. Here the gauge-fixing term $(\partial \cdot A)=\delta A\left(\delta=-* d *, \delta^{2}=0\right)$ is derived from the co-exterior derivative ${ }^{\ddagger \ddagger} \delta$ which is the 'Hodge dual' of $d$ and (anti-)ghost

\footnotetext{
${ }^{\dagger}$ We shall not mention, in this section, similar properties associated with the 2D self-interacting nonAbelian gauge theory. However, these features can be seen in detail in $[33,34,40,42]$.

$\ddagger$ The one-form $A=A_{\mu} d x^{\mu}$ defines the vector potential for the Abelian gauge theory. The zero-form
} 
fields $(\bar{C}) C$ are anti-commuting $\left(\bar{C}^{2}=C^{2}=0, C \bar{C}+\bar{C} C=0\right)$ fields which are required to maintain the unitarity and "quantum" gauge invariance (i.e. BRST invariance) together at any arbitrary order of perturbation theory. The above Lagrangian density remains invariant (up to a total derivative) under the following on-shell $(\square C=\square \bar{C}=0)$ nilpotent $\left(\tilde{s}_{(a) b}^{2}=0\right)$ (anti-)BRST symmetry transformations $\left(\tilde{s}_{(a) b}\right)\left(\right.$ with $\left.\tilde{s}_{b} \tilde{s}_{a b}+\tilde{s}_{a b} \tilde{s}_{b}=0\right)$ as [31-33]

$$
\begin{array}{lll}
\tilde{s}_{b} A_{\mu}=\partial_{\mu} C & \tilde{s}_{b} C=0 & \tilde{s}_{b} \bar{C}=-i(\partial \cdot A) \\
\tilde{s}_{a b} A_{\mu}=\partial_{\mu} \bar{C} & \tilde{s}_{a b} \bar{C}=0 & \tilde{s}_{a b} C=+i(\partial \cdot A) .
\end{array}
$$

The same Lagrangian density (4.1) is also invariant (modulo some total derivatives) under the following on-shell ( $\square C=\square \bar{C}=0$ ) nilpotent $\left(\tilde{s}_{(a) d}^{2}=0\right.$ ) (anti-)dual BRST symmetry transformations $\tilde{s}_{(a) d}\left(\right.$ with $\left.\tilde{s}_{b} \tilde{s}_{a d}+\tilde{s}_{a d} \tilde{s}_{d}=0\right)$ as [31-33]

$$
\begin{array}{cll}
\tilde{s}_{d} A_{\mu}=-\varepsilon_{\mu \nu} \partial^{\nu} \bar{C} & \tilde{s}_{d} \bar{C}=0 & \tilde{s}_{d} \bar{C}=-i E \\
\tilde{s}_{a d} A_{\mu}=-\varepsilon_{\mu \nu} \partial^{\nu} C & \tilde{s}_{a d} C=0 & \tilde{s}_{a d} C=+i E .
\end{array}
$$

The anti-commutator of these two symmetries (i.e. $\left\{\tilde{s}_{b}, \tilde{s}_{d}\right\}=\left\{\tilde{s}_{a b}, \tilde{s}_{a d}\right\}=\tilde{s}_{w}$ ) leads to the definition of a bosonic symmetry $\left(\tilde{s}_{w}\right)$. There exists a ghost scale symmetry transformation for this theory where only the ghost fields undergo a scale transformation and other fields remain untransformed. Together, the above symmetries obey an algebra that is reminiscent of the algebra obeyed by the de Rham cohomology operators [31-36].

The kinetic energy- and gauge-fixing terms of the Lagrangian density (4.1) can be linearized by introducing the auxiliary fields $\mathcal{B}$ and $B$ as [31-33]

$$
\tilde{\mathcal{L}}_{B}=\mathcal{B} E-\frac{1}{2} \mathcal{B}^{2}+B(\partial \cdot A)+\frac{1}{2} B^{2}-i \partial_{\mu} \bar{C} \partial^{\mu} C
$$

which respects (modulo some total derivatives), the following off-shell nilpotent $\left(\tilde{s}_{(A) B}^{2}=\right.$ $\left.\tilde{s}_{(A) D}^{2}=0\right)\left(\right.$ anti-)BRST $\left(\tilde{s}_{(A) B}\right)$ and (anti-)dual BRST $\left(\tilde{s}_{(A) D}\right)$ symmetry transformations

$$
\begin{array}{llllll}
\tilde{s}_{B} A_{\mu} & =\partial_{\mu} C & \tilde{s}_{B} C=0 & \tilde{s}_{B} \bar{C}=i B & \tilde{s}_{B} B=0 & \tilde{s}_{B} \mathcal{B}=0 \\
\tilde{s}_{A B} A_{\mu}=\partial_{\mu} \bar{C} & \tilde{s}_{A B} \bar{C}=0 & \tilde{s}_{A B} C=-i B & \tilde{s}_{A B} B=0 & \tilde{s}_{A B} \mathcal{B}=0 \\
\tilde{s}_{D} A_{\mu}=-\varepsilon_{\mu \nu} \partial^{\nu} \bar{C} & \tilde{s}_{D} \bar{C}=0 & \tilde{s}_{D} C=-i \mathcal{B} & \tilde{s}_{D} \mathcal{B}=0 & \tilde{s}_{D} B=0 \\
\tilde{s}_{A D} A_{\mu}=-\varepsilon_{\mu \nu} \partial^{\nu} C & \tilde{s}_{A D} C=0 & \tilde{s}_{A D} \bar{C}=+i \mathcal{B} & \tilde{s}_{A D} \mathcal{B}=0 & \tilde{s}_{A D} B=0 .
\end{array}
$$

Here too, the anti-commutator $\left\{\tilde{s}_{B}, \tilde{s}_{D}\right\}=\left\{\tilde{s}_{A B}, \tilde{s}_{A D}\right\}=\tilde{s}_{W}$ leads to the existence of the off-shell version of a bosonic symmetry and a scale transformation on the ghost fields defines a ghost symmetry. Thus, in the off-shell version too, we have six symmetries in the theory. Corresponding to the equations (3.7) and (3.8), we can write the expressions for the Lagrangian density of the 2D free Abelian gauge theory as

$$
\begin{aligned}
& \tilde{\mathcal{L}}_{B}=\mathcal{B} E-\frac{1}{2} \mathcal{B}^{2}+X \quad \tilde{\mathcal{L}}_{B}=B(\partial \cdot A)+\frac{1}{2} B^{2}+Y \\
& X=\tilde{s}_{B}\left[-i C\left(\partial \cdot A+\frac{1}{2} B\right)\right]=\tilde{s}_{A B}\left[+i C\left(\partial \cdot A+\frac{1}{2} B\right)\right] \\
& Y=\tilde{s}_{A D}\left[-i \bar{C}\left(E-\frac{1}{2} \mathcal{B}\right)\right]=\tilde{s}_{D}\left[+i C\left(E-\frac{1}{2} \mathcal{B}\right)\right] .
\end{aligned}
$$

(gauge-fixing) $\delta A=(\partial \cdot A)$ and the curvature 2-form (field strength tensor) $F^{(A)}=d A$ are 'Hodge dual' to each-other in any arbitrary dimension of spacetime. Here $\delta= \pm * d *$ is the co-exterior derivative w.r.t. $d$. The same is not true $\left(F^{(N)} \neq d A\right)$ for the non-Abelian gauge theory (see, e.g. [26-30]). 
Moreover, modulo some total derivatives, the Lagrangian density (4.1) can be expressed as the following sum of the (anti-)BRST and (anti-)co-BRST parts

$$
\begin{aligned}
& \tilde{\mathcal{L}}_{b}=\tilde{s}_{d}\left(\frac{i}{2} E C\right)-\tilde{s}_{b}\left(\frac{i}{2}(\partial \cdot A) \bar{C}\right) \equiv\left\{\tilde{Q}_{d}, \frac{1}{2} E C\right\}-\left\{\tilde{Q}_{b}, \frac{1}{2}(\partial \cdot A) \bar{C}\right\} \\
& \tilde{\mathcal{L}}_{b}=\tilde{s}_{a b}\left(\frac{i}{2}(\partial \cdot A) C\right)-\tilde{s}_{a d}\left(\frac{i}{2} E \bar{C}\right) \equiv\left\{\tilde{Q}_{a b}, \frac{1}{2}(\partial \cdot A) C\right\}-\left\{\tilde{Q}_{a d}, \frac{1}{2} E \bar{C}\right\}
\end{aligned}
$$

where conserved and on-shell nilpotent (anti-)co-BRST charges $\tilde{Q}_{(a) d}$ and (anti-)BRST charges $\tilde{Q}_{(a) b}$ are the generators for the on-shell nilpotent (co-)BRST symmetry transformations $\tilde{s}_{(a) d}$ and $\tilde{s}_{(a) b}$. The local expressions for these charges can be found in [31-36]. We do not need these expressions for our present discussions here. A close look at (4.6) and (4.7) implies that equation (4.7) for the Lagrangian density in (4.1) can be derived from (4.6) by exploiting the equations of motion $B=-(\partial \cdot A), \mathcal{B}=E$ and substituting these in the expressions for $X$ and $Y$. The expression for the Lagrangian density in (4.7) implies that the symmetric energy-momentum tensor $T_{\mu \nu}$ for the theory would also be the sum of (anti-)BRST and (anti-)co-BRST invariant parts [33,37]. As a consequence, both the Lagrangian density as well as the symmetric energy momentum tensor would be sum of (anti-)BRST and (anti-)co-BRST anti-commutators. In fact, the set of conserved charges $\left(Q_{b}, Q_{d}, Q_{w}\right)$ (or $\left.Q_{a d}, Q_{a b}, Q_{w}\right)$ can be exploited to define the Hodge decomposition of a state with ghost number $n$ (i.e. $i Q_{g}\left|\psi>_{n}=n\right| \psi>_{n}$ ) in the quantum Hilbert space as

$$
\left|\psi>_{n}=\right| \omega>_{n}+Q_{b}\left|\theta>_{n-1}+Q_{d}\right| \chi>_{n+1} \equiv\left|\omega>_{n}+Q_{a d}\right| \theta>_{n-1}+Q_{a b} \mid \chi>_{n+1}
$$

where $\mid \omega>_{n}$ is the harmonic state, $Q_{b} \mid \theta>_{n-1}$ is the BRST exact state and $Q_{d} \mid \chi>_{n+1}$ is the co-BRST exact state. The other states, in the above equation, can be defined similarly. The choice of the harmonic state $\left(Q_{b} \mid\right.$ phys $>=0, Q_{d} \mid$ phys $\left.>=0\right)$ to be the physical state of the theory furnishes a proof for the topological nature of the theory because there is no energy excitation (i.e. $<$ phys $\left|\hat{T}_{00}\right|$ phys $>^{\prime}=0$ ) in the physical sector of the theory [33,38-41] as the symmetric energy momentum tensor is the sum of (co-)BRST anti-commutators and the conserved, nilpotent and hermitian (co-)BRST charges $Q_{(d) b}$ satisfy $Q_{b} \mid$ phys $>=0=<$ phys $\left|Q_{b}, Q_{d}\right|$ phys $>=0=<$ phys $\mid Q_{d}$.

Now we wish to dwell a bit on (3.7) and study some of the properties of the 2-form antisymmetric Abelian gauge theory in 4D treating our discussions on the one-form 2D free Abelian gauge theory as the backdrop. In fact, the similarity between (4.6) and (3.7) propel us to look into this aspect. Exploiting the equations of motion of (2.7), it can be checked that, modulo some total derivatives, $G$ and $F$ of (3.8) can be re-expressed as

$$
\begin{aligned}
G \rightarrow G^{(0)} & =-\frac{1}{4} C^{\mu}\left(\varepsilon_{\mu \nu \kappa \zeta} \partial^{\nu} B^{\kappa \zeta}\right)-\frac{1}{2}(\partial \cdot \bar{C}) \beta \\
F \rightarrow F^{(0)} & =+\frac{1}{2} \bar{C}^{\mu}\left(\partial^{\nu} B_{\nu \mu}\right)+\frac{1}{2}(\partial \cdot C) \bar{\beta}
\end{aligned}
$$

Now following the analogy with the 2D free Abelian one-form gauge theory (discussed above), it can be checked that

$$
\begin{aligned}
& s_{d}\left(G^{(0)}\right)+s_{b}\left(F^{(0)}\right)=\tilde{\mathcal{L}}_{b}^{(0)}+\partial_{\mu}\left[L^{\mu}\right] \\
& L^{\mu}=\frac{1}{2}\left[\partial^{\mu}(\beta \bar{\beta})-\left(\partial^{\mu} \bar{C}^{\nu}-\partial^{\nu} \bar{C}^{\mu}\right) C_{\nu}-\bar{C}_{\nu}\left(\partial^{\mu} C^{\nu}-\partial^{\nu} C^{\mu}\right)\right]
\end{aligned}
$$


where the Lagrangian density $\tilde{\mathcal{L}}_{b}^{(0)}$

$$
\begin{aligned}
\tilde{\mathcal{L}}_{b}^{(0)} & =\frac{1}{2}\left(\partial^{\kappa} B_{\kappa \mu}-\partial_{\mu} \phi_{1}\right)\left(\partial_{\sigma} B^{\sigma \mu}\right)-\frac{1}{2}\left(\frac{1}{2} \varepsilon_{\mu \nu \kappa \zeta} \partial^{\nu} B^{\kappa \zeta}-\partial_{\mu} \phi_{2}\right)\left(\frac{1}{2} \varepsilon^{\mu \xi \sigma \eta} \partial_{\xi} B_{\sigma \eta}\right) \\
& -\partial_{\mu} \bar{\beta} \partial^{\mu} \beta+\left(\partial_{\mu} \bar{C}_{\nu}-\partial_{\nu} \bar{C}_{\mu}\right)\left(\partial^{\mu} C^{\nu}\right)-\frac{1}{2}(\partial \cdot \bar{C})(\partial \cdot C)
\end{aligned}
$$

is different from the Lagrangian density (2.8) that respects the on-shell nilpotent (anti)BRST and (anti-)co-BRST symmetries. Even though the ghost part of the above Lagrangian density is exactly same as in (2.8), there are some terms in the bosonic part of (2.8) that are absent here. For instance, it can be checked that the terms in (4.10), that contain the auxiliary scalar fields $\phi_{1}, \phi_{2}$, are total derivatives. Thus, they can be dropped from the total action as they do not influence dynamics of the theory in any way. As a consequence, the above Lagrangian density in (4.10) can be treated to be independent of the terms containing fields $\phi_{1}$ and $\phi_{2}$ which are very much present in (2.8). It will be noted that this feature of the 2-form 4D Abelian gauge theory is drastically different from the corresponding 2D one-form Abelian gauge theory (which we have discussed above). The upshot of our whole discussion is the fact that the Lagrangian density in (2.8) (that respects on-shell nilpotent (anti-)BRST and (anti-)co-BRST symmetries) cannot be expressed as the sum of (anti-) BRST and (anti-)co-BRST anti-commutators as is the case for the 2D free Abelian (cf (4.7)) and self-interacting non-Abelian gauge theories [33,34,40,42].

Now let us take a different choice in place of $F^{(0)}$ and $G^{(0)}$ so that we can get the correct bosonic part of the Lagrangian density (2.8). An interesting, such a choice, at our disposal is

$$
F^{(1)}=F^{(0)}+\frac{1}{2}(\partial \cdot \bar{C}) \phi_{1} \quad G^{(1)}=G^{(0)}-\frac{1}{2}(\partial \cdot C) \phi_{2} .
$$

The variation of these expressions w.r.t. the on-shell nilpotent (co-)BRST transformations can be written explicitly as

$$
\begin{aligned}
s_{b}\left(F^{(1)}\right) & =\frac{1}{2}\left(\partial^{\nu} B_{\nu \mu}-\partial_{\mu} \phi_{1}\right)\left(\partial_{\sigma} B^{\sigma \mu}-\partial^{\mu} \phi_{1}\right)-\frac{1}{2} \partial_{\mu} \bar{\beta} \partial^{\mu} \beta \\
& +\frac{1}{2}\left(\partial_{\mu} \bar{C}_{\nu}-\partial_{\nu} \bar{C}_{\mu}\right)\left(\partial^{\mu} C^{\nu}\right)-\frac{1}{2}(\partial \cdot \bar{C})(\partial \cdot C)+\partial_{\mu} M^{\mu} \\
s_{d}\left(G^{(1)}\right) & =-\frac{1}{2}\left(\frac{1}{2} \varepsilon_{\mu \nu \kappa \zeta} \partial^{\nu} B^{\kappa \zeta}-\partial_{\mu} \phi_{2}\right)\left(\frac{1}{2} \varepsilon^{\mu \xi \sigma \eta} \partial_{\xi} B_{\sigma \eta}-\partial^{\mu} \phi_{2}\right) \\
& -\frac{1}{2} \partial_{\mu} \beta \partial^{\mu} \beta+\frac{1}{2}\left(\partial_{\mu} \bar{C}_{\nu}-\partial_{\nu} \bar{C}_{\mu}\right)\left(\partial^{\mu} C^{\nu}\right)-\frac{1}{2}(\partial \cdot \bar{C})(\partial \cdot C)+\partial_{\mu} N^{\mu}
\end{aligned}
$$

where the expressions for the $M^{\mu}$ and $N^{\mu}$ in terms of the local fields are

$$
\begin{aligned}
M^{\mu} & =\frac{1}{2}\left[\bar{\beta} \partial^{\mu} \beta-\bar{C}_{\nu}\left(\partial^{\mu} C^{\nu}-\partial^{\nu} C^{\mu}\right)+\left(\partial_{\nu} B^{\nu \mu}-\partial^{\mu} \phi_{1}\right) \phi_{1}\right] \\
N^{\mu} & =\frac{1}{2}\left[\beta \partial^{\mu} \bar{\beta}-\left(\partial^{\mu} \bar{C}^{\nu}-\partial^{\nu} \bar{C}^{\mu}\right) C_{\nu}+\left(\frac{1}{2} \varepsilon^{\mu \nu \kappa \zeta} \partial_{\nu} B_{\kappa \zeta}-\partial^{\mu} \phi_{2}\right) \phi_{2}\right] .
\end{aligned}
$$

The sum of (4.11) and (4.12) yields, modulo some total derivatives, the Lagrangian density of (2.8) but for the last term as

$$
\begin{aligned}
\mathcal{L}_{b}^{(0)} & =\partial_{\mu}\left(P^{\mu}\right)+\frac{1}{2}\left(\partial^{\nu} B_{\nu \mu}-\partial_{\mu} \phi_{1}\right)\left(\partial_{\sigma} B^{\sigma \mu}-\partial^{\mu} \phi_{1}\right) \\
& -\frac{1}{2}\left(\frac{1}{2} \varepsilon_{\mu \nu \kappa \zeta} \partial^{\nu} B^{\kappa \zeta}-\partial_{\mu} \phi_{2}\right)\left(\frac{1}{2} \varepsilon^{\mu \xi \sigma \eta} \partial_{\xi} B_{\sigma \eta}-\partial^{\mu} \phi_{2}\right) \\
& -\partial_{\mu} \beta \partial^{\mu} \beta+\left(\partial_{\mu} \bar{C}_{\nu}-\partial_{\nu} \bar{C}_{\mu}\right)\left(\partial^{\mu} C^{\nu}\right)-\left(\frac{\mathbf{1}}{\mathbf{2}}+\frac{\mathbf{1}}{\mathbf{2}}\right)(\partial \cdot \bar{C})(\partial \cdot C)
\end{aligned}
$$

where the local expression for $P^{\mu}$ is

$$
\begin{aligned}
P^{\mu} & =\frac{1}{2}\left[\partial^{\mu}(\bar{\beta} \beta)-\bar{C}_{\nu}\left(\partial^{\mu} C^{\nu}-\partial^{\nu} C^{\mu}\right)+\left(\partial_{\nu} B^{\nu \mu}-\partial^{\mu} \phi_{1}\right) \phi_{1}\right. \\
& \left.-\left(\partial^{\mu} \bar{C}^{\nu}-\partial^{\nu} \bar{C}^{\mu}\right) C_{\nu}+\left(\frac{1}{2} \varepsilon^{\mu \nu \kappa \zeta} \partial_{\nu} B_{\kappa \zeta}-\partial^{\mu} \phi_{2}\right) \phi_{2}\right] .
\end{aligned}
$$


Thus, we note that except for an extra factor of half in the last term, the Lagrangian density for the 4D 2-form Abelian gauge theory can be written as the sum of (anti-)BRST and (anti-)co-BRST anti-commutators. It is evident now that the symmetric energy momentum for the theory will also not be able to be expressed as the sum of two anti-commutators. Hence, the free 2-form Abelian gauge theory in 4D is not an example of an exact TFT but (i) it is a field theoretical model for the Hodge theory in 4D, and (ii) its properties are such that it can be treated as an example of a field theoretical model for the q-TFT.

\section{Conclusions}

In the present investigation, we studied the free 4D Abelian 2-form (antisymmetric) gauge theory from the point of view of (i) its cohomological properties, and (ii) its quasitopological nature. In the first part, we invoked some of the results of our earlier work [25] because these inferences and issues were pertinent and complementary to our present work. We found that the above theory provides a tractable field theoretical model for the Hodge theory in the physical four $(3+1)$-dimensions of spacetime. The symmetries of the Lagrangian density correspond to all the de Rham cohomological operators of differential geometry. In fact, there are six local, covariant and continuous symmetries in the theory of which four $\left(s_{(A) B}, s_{(A) D}\right)$ symmetries are nilpotent $\left(s_{(A) B}^{2}=s_{(A) D}^{2}=0\right)$ of order two. The latter symmetries are very interesting and they play a pivotal role in expressing the "topological" invariants of the theory which obey a certain specific type of recursion relations. Moreover, these symmetries and their anticommutator (which corresponds to a bosonic symmetry) obey an algebra that is also respected by the corresponding conserved Noether charges $Q_{r}(r=A B, B, A D, D, W)$ for the off-shell version of the symmetries [25]

$$
\begin{array}{lll}
Q_{(A) B}^{2} & =0 & Q_{(A) D}^{2}=0 \quad\left\{Q_{B}, Q_{D}\right\}=Q_{W}=\left\{Q_{A B}, Q_{A D}\right\} \\
\left\{Q_{A B}, Q_{B}\right\}=0 & \left\{Q_{D}, Q_{A D}\right\}=0 \quad\left[Q_{W}, Q_{r}\right]=0 .
\end{array}
$$

The above algebra is reminiscent of the algebra obeyed by $(d, \delta, \Delta)$. It will be noted that the nilpotent pair $(d, \delta)$ together can not be identified with $\left(Q_{B}, Q_{A B}\right)$ or $\left(Q_{D}, Q_{A D}\right)$ because the latter pairs of generators anti-commute with each-other in their set whereas the former pair $(d, \delta)$ does not. The algebra among the cohomological operators can be succinctly expressed as

$$
\begin{array}{lcc}
d^{2}=0 & \delta^{2}=0 \quad \Delta=(d+\delta)^{2}=d \delta+\delta d \\
{[\Delta, d]=0} & {[\Delta, \delta]=0} & \Delta=\{d, \delta\} \neq 0
\end{array}
$$

The algebra between conserved charges and the ghost charge $Q_{g}$

$$
i\left[Q_{g}, Q_{B(A D)}\right]=+Q_{B(A D)} \quad i\left[Q_{g}, Q_{D(A B)}\right]=-Q_{D(A B)} \quad i\left[Q_{g}, Q_{W}\right]=0
$$

is such that any arbitrary state $\mid \psi>_{n}$ in the the quantum Hilbert space of states with ghost number $n$ (i.e. $i Q_{g}\left|\psi>_{n}=n\right| \psi>_{n}$ ) would imply the following (see, e.g., [25])

$$
\begin{aligned}
& i Q_{g} Q_{B(A D)}\left|\psi>_{n}=(n+1) Q_{B(A D)}\right| \psi>_{n} \\
& i Q_{g} Q_{D(A B)}\left|\psi>_{n}=(n-1) Q_{D(A B)}\right| \psi>_{n} \\
& i Q_{g} Q_{W}\left|\psi>_{n}=n Q_{W}\right| \psi>_{n} .
\end{aligned}
$$


This shows that the ghost numbers for the states $Q_{B(A D)}\left|\psi>_{n}, Q_{D(A B)}\right| \psi>_{n}$ and $Q_{W} \mid \psi>_{n}$ in the quantum Hilbert space are $(n+1),(n-1)$ and $n$, respectively. This feature is same as the result of the application of the cohomological operators $(d, \delta, \Delta)$ on a differential form $f_{n}$ of degree $n$. This analogy is valid due to the fact that the forms $d f_{n}, \delta f_{n}$ and $\Delta f_{n}$ possess the degree $n+1, n-1$ and $n$, respectively. This discussion, allows us to find a mapping between cohomological operators and conserved charges as: $d \leftrightarrow Q_{B(A D)}, \delta \leftrightarrow Q_{D(A B)}, \Delta \leftrightarrow Q_{W}$. This establishes the fact that $4 \mathrm{D}$ free Abelian 2-form gauge theory is a field theoretical model for the Hodge theory because the discrete transformations in (2.4) correspond to the Hodge duality $*$ operation of the differential geometry.

Now we wish to state some of the key results of the second part of our investigation. We focussed on some of the distinguished properties of the 4D 2-form Abelian gauge theory vis-à-vis 2D topological Abelian (as well as self-interacting non-Abelian) one-form gauge theory. We observed some common features and a few striking differences between these theories. The overlapping similarities are: (i) the form of the Lagrangian densities of both the theories can be expressed in the same fashion (cf eqns (3.7) and (4.6)). It should be emphasized, however, that the above statement is found to be true only when we exploit the off-shell nilpotent version of the (anti-)BRST and (anti-)co-BRST symmetries. (ii) We could derive "topological invariant" quantities on the 4D manifold that are similar to the topological invariants of the 2D one-form (non-)Abelian gauge theories defined on the 2D manifold [31-34,38-43]. (iii) These invariants obey the same kind of recursion relations as invariants of topological field theories. (iv) Both the theories (i.e. 4D 2-form and 2D oneform free Abelian gauge theories) provide a set of tractable field theoretical models for the Hodge theory. (v) The Lagrangian densities of both the theories respect six local, covariant and continuous symmetries. Now, we dwell a bit on the key differences between these two theories. These are: (i) as far as the form and appearance of the Lagrangian densities (and symmetric energy-momentum tensor) are concerned, there exists a slight difference between the two theories when they are expressed in terms of the on-shell version of the nilpotent symmetries. It is clear from (4.7) that the Lagrangian density (so the symmetric energy momentum tensor) for the 2D free Abelian (and self-interacting non-Abelian) gauge theories can be expressed, modulo some total derivatives, as the sum of (anti-)BRST and (anti-)co-BRST anti-commutators. This is not the case with the 4D 2-form Abelian gauge theory as can be seen from (4.14). In fact, the Lagrangian density (and therefore the symmetric energy-momentum tensor) for the latter theory cannot be exactly expressed as the sum of (anti-)BRST and (anti-)co-BRST anti-commutators. The two theories mathematically resemble very much but for a factor of half. (ii) The free 2D (non-)Abelian gauge theories, defined on the 2D manifold without a non-trivial topology at the boundary, are topological in nature but the same is not true for the 4D 2-form Abelian gauge theory. In fact, the counting of degrees of freedom also shows it. However, some of the striking similarities between these two theories propel us to conclude that 4D 2-form gauge theory is possibly an example of the field theoretical models for the q-TFT. (iii) The topological 
invariants $I_{k}\left(\tilde{I}_{k}\right)$ and $J_{k}\left(\tilde{J}_{k}\right)$ for the $2 \mathrm{D}$ (non-)Abelian theories are defined on the $2 \mathrm{D}$ closed Riemann surface (i.e. the Eauclidean version [51] of the 2D manifold) for all the possible values of $k=0,1,2$. The analogous thing does not happen for the present 4D 2-form gauge theory because all the invariants do not exist for all the possible values of $k=0,1,2,3,4$. At least, in our present investigation, we are unable to provide a logical reason behind this discrepancy. Perhaps, this difference too corroborates the non-topological nature of 4D 2-form gauge theory. (iv) There exist four sets of topological invariants w.r.t. (anti-)BRST and (anti-)co-BRST charges of the 2D theories because of the fact that there is a single set of fermionic scalar (anti-)ghost fields $(\bar{C}) C$. This is not the case with 4D 2-form Abelian gauge theory. In fact, for the latter theory, we have a single set of fermionic vector (anti)ghost fields $\left(\bar{C}_{\mu}\right) C_{\mu}$, a single set of fermionic scalar (anti-)ghost fields $(\rho) \lambda$ and a single set of bosonic (anti-)ghost fields $(\bar{\beta}) \beta$. Therefore, four sets of invariants exist for each of these sets w.r.t. (anti-)BRST and (anti-)co-BRST charges which makes the total number of invariants to be twelve. These have been computed in section 3 of our present paper.

\section{Acknowledgements}

Constructive, clarifying and critical comments by the referees' are gratefully acknowledged. Thanks are also due to E. Harikumar and A. Lahiri for taking interest in this work.

\section{References}

[1] Ogievetsky V I and Palubarinov I V 1967 Sov. J. Nucl. Phys. (Iad. Fiz.) 4156

[2] Deser S 1969 Phys. Rev. 1871931

[3] Aurilia A and Takahashi Y 1981 Prog. Theor. Phys. 66693

[4] See, e.g., Salam A and Sezgin E 1989 Supergravities in Diverse Dimensions (Singapore: World Scientific)

[5] Green M B, Schwarz J H and Witten E 1987 Superstring Theory (Cambridge: Cambridge University Press)

[6] Polchinski J 1998 String Theory (Cambridge: Cambridge University Press)

[7] See, e.g., Seiberg N and Witten E 1999 J. High Energy Phys. 09032

[8] Kalb M and Ramond P 1974 Phys. Rev. D 92273

[9] Vilenkin A and Vachaspati T 1987 Phys. Rev. D 351138

[10] Davis R L 1989 Phys. Rev. D 404033

Davis R L 1990 Mod. Phys. Lett. A 5955

[11] Allen T J, Bowick M J and Lahiri A 1989 Phys. Lett. B 23747 
[12] Cremmer E and Scherk J 1974 Nucl. Phys. B 72117

Oda I and Yahokozawa S 1990 Prog. Theor. Phys. 83991

Deguchi S, Mukai and T Nakajima T 1999 Phys. Rev. D 5965003

[13] Kaul R K 1978 Phys. Rev. D 181127

[14] See, e.g., Lahiri A 1993 Mod. Phys. Lett. A 82403

[15] See, e.g., Harikumar E and Sivakumar M 2000 Nucl. Phys. B 565385

[16] See, e.g., Lahiri A 1997 Phys. Rev. D 555045

[17] See, e.g., Harikumar E and Sivakumar M 1998 Phys. Rev. D 573794

[18] See, e.g., Lahiri A 1997 Mod. Phys. Lett. A 122699

[19] Townsend P K 1979 Phys. Lett. B 8897

[20] Henneaux M and Teitelboim C 1992 Quantization of Gauge Systems (Princeton NJ: Princeton University Press)

[21] Gomis J, Paris J and Samuel S 1995 Phys. Rep. 2591

[22] Kimura T 1980 Prog.Theor. Phys. 64357

[23] Thierry-Mieg J and Baulieu L 1983 Nucl. Phys. B 228259

[24] Barcelos-Neto J and Thibes R 1998 J. Math. Phys. 395669

[25] Harikumar E, Malik R P and Sivakumar M 2000 J. Phys. A: Math Gen 337149 (Preprint hep-th/0004145)

[26] Eguchi T, Gilkey P B and Hanson A J 1980 Phys. Rep. 66213

[27] Mukhi S and Mukunda N 1990 Introduction to Topology, Differential Geometry and Group Theory for Physicists (New Delhi: Wiley Eastren)

[28] Nishijima K 1988 Prog. Theor. Phys. 80897

Nishijima K 1988 Prog. Theor. Phys. 80905

[29] van Holten J W 1990 Phys. Rev. Lett. 642863

van Holten J W 1990 Nucl. Phys. B 339158

Aratyn H 1990 J. Math. Phys. 311240

[30] Fülöp G and Marnelius R 1995 Nucl. Phys. B 456442

Kalau W and van Holten J W 1991 Nucl. Phys. B 361233

[31] Malik R P 2000 Int. J. Mod. Phys. A 151685 (Preprint hep-th/9808040)

[32] Malik R P 2000 J. Phys. A: Math Gen 332437 (Preprint hep-th/9902146)

[33] Malik R P 2001 J. Phys. A: Math Gen 344167 (Preprint hep-th/0012085)

[34] Malik R P 1999 Mod. Phys. Lett. A 141937 (Preprint hep-th/9903121)

[35] Malik R P 2000 Mod. Phys. Lett. A 152079 (Preprint hep-th/0003128)

[36] Malik R P 2001 Mod. Phys. Lett. A 16477 (Preprint hep-th/9711056) 
[37] For review, see, e.g., Birmingham D, Blau M, Rakowski M and Thompson G 1991 Phys. Rep. 209129

[38] Malik R P 2002 J. Phys. A: Math Gen 353711 (Preprint hep-th/0106215)

[39] Malik R P 2002 J. Phys. A: Math Gen 356919 (Preprint hep-th/0112260)

[40] Malik R P 2002 J. Phys. A: Math Gen 358817 (Preprint hep-th/0204015)

[41] Malik R P 2002 Cohomological aspects of gauge theories: superfield formalism (Preprint hep-th/0205135)

To appear in: 2003 Ann. Phys. (N. Y.)

[42] Malik R P 2002 Mod. Phys. Lett. A 17185 (Preprint hep-th/0111253)

[43] Malik R P 2001 Phys. Lett. B 521409 (Preprint hep-th/0108105)

[44] Witten E 1988 Commun. Math. Phys. 117353

Witten E 1989 Commun. Math. Phys. 121351

[45] Schwarz A S 1978 Lett. Math. Phys. 2247

[46] Schaller P and Strobl T 1994 Mod. Phys. Lett. A 93129

Schaller P and Strobl T 1996 Lecture Notes in Phys. 469321

See also: Schaller P and Strobl T 1994 Poisson $\sigma$-models: A generalization of 2D gravity-Yang-Mills systems (Preprint hep-th/9411163)

[47] Weinberg S 1996 The quantum theory of fields: Modern applications vol 2 (Cambridge: Cambridge University Press)

[48] Deser S, Gomberoff A, Henneaux M and Teitelboim C 1997 Phys. Lett. B 40080

[49] Banerjee R and Wotzasek C 1998 Nucl. Phys. B 527402

[50] Banerjee R and Chakraborty B 1999 J. Phys. A: Math Gen 324441

[51] Soda J 1991 Phys. Lett. B 267214

Hosoya A and Soda J 1989 Mod. Phys. Lett. A 42539

Abe M and Nakanishi N 1993 Prog. Theor. Phys. 89501

[52] Nakanishi N and Ojima I 1990 Covariant Operator Formalism of Gauge Theories and Quantum Gravity (Singapore: World Scientific)

[53] Nishijima K 1986 in: Progress in Quantum Field Theory Eds. Ezawa H and Kamefuchi S (Amsterdam: North-Holland) 